\title{
Atomic and wavelet characterization of Musielak-Orlicz Hardy spaces for generalized Orlicz functions
}

Mitsuo Izuki, Eiichi Nakai and Yoshihiro Sawano

\begin{tabular}{|c|l|}
\hline Citation & OCAMI Preprint Series. 2021, 21-2. \\
\hline Issue Date & $2021-05-20$ \\
\hline Type & Preprint \\
\hline Textversion & Author \\
\hline Rights & For personal use only. No other uses without permission. \\
\hline Relation & $\begin{array}{l}\text { This is a pre-print of an article published in Integral Equations and } \\
\text { Operator Theory. The final authenticated version is available online at: } \\
\text { https://doi.org/10.1007/s00020-021-02672-2 }\end{array}$ \\
\hline
\end{tabular}

From: Osaka City University Advanced Mathematical Institute http://www.sci.osaka-cu.ac.jp/OCAMI/publication/preprint/preprint.html 


\title{
Atomic and wavelet characterizations of Musielak-Orlicz Hardy spaces for generalized Orlicz functions
}

\author{
Mitsuo Izuki, Eiichi Nakai and Yoshihiro Sawano
}

May 20, 2021

\begin{abstract}
The goal of this paper is to obtain atomic and wavelet characterizations of Musielak-Orlicz Hardy spaces. Recently, in 2018, Fu and Yang obtained wavelet characterizations of Musielak-Orlicz Hardy spaces for growth functions of uniformly upper type $p_{-}$and of uniformly lower type $p_{+}$with $0<p_{-} \leq p_{+} \leq 1$. What is different from the existing works is that we merely assume $0<p_{-} \leq p_{+}<\infty$. One of the important tools that make it possible is to refine the convexity of Orlicz functions by obtaining canonical equivalent functions. As applications of the atomic characterization, we investigate the boundedness property of singular integral operators. Especially, we obtain the boundedness property of Marcinkiewicz integral operators acting on some Musielak-Orlicz Hardy spaces which are quasi-Banach spaces.
\end{abstract}

Key words: Musielak-Orlicz Hardy space, wavelet characterization, atomic characterization, Marcinkiewicz operator, singular integral operator.

AMS Subject Classification: 42B35, 42C40.

\section{Introduction}

The goal of this paper is to obtain atomic and wavelet characterizations of MusielakOrlicz Hardy spaces generated by a generalized Musielak-Orlicz function of uniformly lower type and uniformly upper type. What is new in this paper is that we can deal with generalized Musielak-Orlicz functions of uniformly lower type $p_{-}$ and uniformly upper type $p_{+}$for any $0<p_{-} \leq p_{+}<\infty$, while many earlier works considered the case where $0<p_{-} \leq p_{+} \leq 1$.

Hardy spaces associated with Musielak-Orlicz spaces seems to go back to [17]. Ky [17] introduced the Musielak-Orlicz Hardy space $H^{\varphi}\left(\mathbb{R}^{n}\right)$, which extends Orlicz Hardy spaces and weighted Hardy spaces. Here by a weight we mean a non-negative measurable function, although we will postulate more conditions on weights later. Among other function spaces, Musielak-Orlicz Hardy space has attracted much attention. For example, the Musielak-Orlicz Hardy space $H^{\theta}\left(\mathbb{R}^{n}\right)$ generated by 
the function

$$
\theta(x, t)=\frac{t}{\log (e+|x|)+\log (e+t)} \quad\left((x, t) \in \mathbb{R}^{n}\right)
$$

turned out to be important in connection with pointwise multipliers on $\operatorname{BMO}\left(\mathbb{R}^{n}\right)$ and $H^{1}\left(\mathbb{R}^{n}\right)$. This remarkable property of $\theta$ is pointed out by $\mathrm{Ky}$ [17], who is motivated by the result of the pointwise multiplier obtained by the second author and Yabuta [24].

One of the convenient ways to develop the theory of Hardy spaces associated with Banach lattices is to consider the duality. As in [25], the duality of a Banach lattice together with the boundedness property of the Hardy-Littlewood maximal operator allows us to investigate the Hardy space associated to this lattice with ease. The technique in [25], whose ingredient is the duality, can be applied to many settings such as Morrey spaces [14], local Morrey spaces [3], Morrey spaces with variable exponent [10], weak Lebesgue spaces [30], generalized Morrey spaces [1], local Morrey type spaces [8] and mixed Lebesgue spaces [11]. However, this does not apply to Musielak-Orlicz spaces because we can not handle the FenchelLegendre transform of the (Musielak-)Orlicz functions, namely, we can not employ the technique in [25]. To overcome this problem, we will go through a similar argument to [23]. To this end, we will prove an estimate based on the notions of strictly uniformly lower type and strictly uniformly upper type. Unfortunately, as in [31, Remark 1.7(iv)] that the Musielak-Orlicz Hardy space and the variable exponent Hardy space cannot cover each other. Thus, we need to establish the theory of Musielak-Orlicz Hardy spaces from scratch.

Here we survey the history of the wavelet characterization of Hardy spaces including related characterizations. Meyer [20] established several equivalent wavelet characterizations of $H^{1}\left(\mathbb{R}^{n}\right)$. Liu [19] established some equivalent wavelet characterizations of the weak Hardy space $H^{1, \infty}\left(\mathbb{R}^{n}\right)$. Wu [33] obtained further a wavelet area integral characterization of the weighted Hardy space $H^{p}\left(\mathbb{R}^{n}\right)$ for any $p \in(0,1]$ and later, García-Cuerva and Martell [6] established a characterization of $H^{p}\left(\mathbb{R}^{n}\right)$ for any $p \in(0,1]$ in terms of wavelets without compact supports using the vectorvalued Calderón-Zygmund theory. Kopaliani [16] and Izuki [12] independently introduced the wavelet inequalities of Lebesgue spaces with variable exponents. The present authors [13] further obtained the wavelet characterization for weighted Lebesgue spaces with variable exponents. Fu and Yang obtained the wavelet characterization for Musielak-Orlicz Hardy spaces in [5]. Maeda and Shimomura [21] investigated Hardy spaces associated with Musielak-Orlicz spaces together with the third author. See [29] for more.

Here we explain the notation which we employ in this paper.

- Let $\mathbb{N}_{0} \equiv\{0,1, \ldots\}$.

- We write $\mathbb{R}_{+}^{n+1} \equiv \mathbb{R}^{n} \times(0, \infty)$.

- A set $S$ is said to be a dyadic cube if

$$
S=Q_{j, k} \equiv \prod_{m=1}^{n}\left[2^{-j} k_{m}, 2^{-j}\left(k_{m}+1\right)\right]
$$


for some $j \in \mathbb{Z}$ and $k=\left(k_{1}, k_{2}, \ldots, k_{n}\right) \in \mathbb{Z}^{n}$. A dyadic cube $S^{\prime}$ with respect to a cube $S$ of the form $S=a Q_{0,0}+b,(b, a) \in \mathbb{R}_{+}^{n+1}$, is a subset $S^{\prime}=a Q_{j, k}+b$ for some $j \in \mathbb{N}_{0}$ and $k \in\left\{0,1, \ldots, 2^{j}-1\right\}^{n}$.

- Let $A, B \geq 0$. Then $A \lesssim B$ and $B \gtrsim A$ mean that there exists a constant $C>0$ such that $A \leq C B$, where $C$ depends only on the parameters of importance. The symbol $A \sim B$ means that $A \lesssim B$ and $B \lesssim A$ happen at the same time, while $A \simeq B$ means that there exists a constant $C>0$ such that $A=C B$.

- The symbols $\mathcal{F}$ and $\mathcal{F}^{-1}$ stand for the Fourier transform and its inverse, respectively. More precisely, for $f \in L^{1}\left(\mathbb{R}^{n}\right)$, define the Fourier transform and the inverse Fourier transform by

$$
\mathcal{F} f(\xi) \equiv(2 \pi)^{-\frac{n}{2}} \int_{\mathbb{R}^{n}} f(x) \mathrm{e}^{-\mathrm{i} x \cdot \xi} \mathrm{d} x, \quad \mathcal{F}^{-1} f(x) \equiv(2 \pi)^{-\frac{n}{2}} \int_{\mathbb{R}^{n}} f(\xi) \mathrm{e}^{\mathrm{i} x \cdot \xi} \mathrm{d} \xi .
$$

The remaining part of this paper is organized as follows: Section 2 recalls some elementary facts on generalized Musielak-Orlicz functions. In addition to the notion of uniformly lower type and uniformly upper type, we define the ones of strictly uniformly lower type and strictly uniformly upper type and then give a lemma on this notion; see Lemma 2.8 to follow. We imitate the idea of [17] to consider the convex inequality in Lemma 2.7. We use Lemmas 2.7 and 2.8 to investigate the relation between the atomic decomposion considered in [17] and the one in this paper; see the latter half of Section 3. Using Lemma 2.7, we obtain an important estimate which parallels the one in [23]; see Lemma 2.16. We define Musielak-Orlicz Hardy spaces and give the atomic characterization using Lemma 2.16 in Section 3. We show the boundedness properties of (generalized) singular integral operators in Section 4 together with the Littlewood-Paley characterization. As a further application of the boundedness results in Section 4, we obtain the wavelet characterization in Section 5. Among others, we justify the coupling $\left\langle f, \psi^{l}\right\rangle$ for the wavelet $\psi^{l} \in C^{s+1}\left(\mathbb{R}^{n}\right)$ and $f \in H^{\varphi}\left(\mathbb{R}^{n}\right) \subset \mathcal{S}^{\prime}\left(\mathbb{R}^{n}\right)$ in Lemma 5.4. Section 6 is oriented to the application of the atomic characterization to generalized Marcinkiewicz integral operators. Usually, when we investigate the boundedness property of generalized Marcinkiewicz integral operators, we use the complex interpolation, which usually forces us to work in Banach spaces. In this paper, by the use of Lemma 2.16 and the idea of Asami [2], we provide a proof which does not use the complex interpolation. Our result will cover spaces $H^{p}\left(\mathbb{R}^{n}\right)$ and $L^{p}\left(\mathbb{R}^{n}\right)$ with $\frac{2 n}{2 n+1}<p \leq 1$.

\section{Preliminaries}

Here we collect preliminary facts. First we give basic properties on generalized Musielak-Orlicz functions in Subsection 2.1. Next, we consider the boundedness property of the Hardy-Littlewood maximal operator $M$ in Subsection 2.2, where we recall the modular inequality. In Subsection 2.3 we recall the properties of the grand maximal operator including integral estimates based on the books $[7,26]$. 


\subsection{Orlicz functions}

An Orlicz function is a convex homeomorphism on $[0, \infty)$. We recall the definition of generalized Musielak-Orlicz functions with two variables $x$ and $t$ following [17, 29].

Definition 2.1 (Generalized Musielak-Orlicz function).

1. A Musielak-Orlicz function is a function $\varphi: \mathbb{R}^{n} \times[0, \infty) \rightarrow[0, \infty)$ such that $\varphi(x, \cdot)$ is an Orlicz function for each $x \in \mathbb{R}^{n}$ and $\varphi(\cdot, t)$ is a measurable function for each $t \geq 0$. A generalized Musielak-Orlicz function is a function $\varphi: \mathbb{R}^{n} \times[0, \infty) \rightarrow[0, \infty)$ such that $\varphi\left(\cdot,{ }^{1 / p}\right): \mathbb{R}^{n} \times[0, \infty) \rightarrow[0, \infty)$ is a Musielak-Orlicz function for some $0<p<\infty$.

2. Let $0<p<\infty$. A generalized Musielak-Orlicz function $\varphi$ is said to be of uniformly lower- (resp., upper-) type $p$ if for any $x \in \mathbb{R}^{n}, t \in[0, \infty)$ and $s \in(0,1)$ (resp., $s \in[1, \infty)), \varphi(x, s t) \lesssim s^{p} \varphi(x, t)$ with the implicit constant independent of $s, t$ and $x$.

3. If $\lesssim$ can replaced by $\leq$ in the above, then $\varphi$ is said to be strictly of uniformly lower- (resp., upper-) type $p$.

4. For a generalized Musielak-Orlicz function $\varphi$ of uniformly lower type $p_{-}$and of uniformly upper type $p_{+}$with $0<p_{-} \leq p_{+}<\infty$, let

$$
i(\varphi) \equiv \sup \{p \in(0, \infty): \varphi \text { is of uniformly lower type } p\}
$$

and

$$
I(\varphi) \equiv \inf \{p \in(0, \infty): \varphi \text { is of uniformly upper type } p\} .
$$

\section{Example 2.2.}

1. As a prototype case, we consider $\varphi(x, t)=t^{p}$ for $(x, t) \in \mathbb{R}_{+}^{n+1}$. Here $0<p<$ $\infty$. In this case, $I(\varphi)=i(\varphi)=p$

2. The function $\theta$, given by (1.1) and considered by Ky, satisfies

$$
I(\theta)=i(\theta)=1 .
$$

It is easy to check that $I(\theta)=1$. To verify that $i(\theta)=1$, we will use

$$
\log (e+t) \geq \log (e+s t) \geq \log \left(e+t^{1-a}\right) \sim \log (e+t)
$$

for any $a \geq 1, s \in[0,1]$ and $t \in[1, \infty)$ with $s t^{a} \geq 1$.

It turns out important that we introduce the notion of strictly of uniformly upper/lower type; see Proposition 2.15.

To define Musielak-Orlicz spaces, we define the class $\mathbb{A}_{q}\left(\mathbb{R}^{n}\right)$ following the work by Ky [17].

\section{Definition 2.3.}


1. $[7,26]$ A locally integrable weight $w$ is said to be an $A_{1}$-weight, if $0<w<$ $\infty$ almost everywhere, and $[w]_{A_{1}} \equiv \sup _{Q \in \mathcal{Q}} m_{Q}(w)\left\|w^{-1}\right\|_{L^{\infty}(Q)}<\infty$, where $\mathcal{Q}$ denotes the set of all cubes in $\mathbb{R}^{n}$.

2. [7, 26] Let $1<q<\infty$. A locally integrable weight $w$ is said to be an $A_{q}$-weight, if $0<w<\infty$ almost everywhere, and

$$
[w]_{A_{q}} \equiv \sup _{Q \in \mathcal{Q}} m_{Q}(w) m_{Q}\left(w^{-\frac{1}{q-1}}\right)^{q-1}<\infty .
$$

3. The class $A_{\infty}$ is defined by $A_{\infty}=\bigcup_{1 \leq q<\infty} A_{q}$.

4. [17] Let $1 \leq q \leq \infty$. A function $\varphi: \mathbb{R}^{n} \times[0, \infty) \rightarrow[0, \infty)$ is said to belong to $\mathbb{A}_{q}\left(\mathbb{R}^{n}\right)$ if $[\varphi]_{\mathbb{A}_{q}}=\sup _{t>0}[\varphi(\cdot, t)]_{A_{q}}<\infty$. A function $\varphi: \mathbb{R}^{n} \times[0, \infty) \rightarrow[0, \infty)$ is said to satisfy the uniformly Muckenhoupt condition if $\varphi \in \mathbb{A}_{q}\left(\mathbb{R}^{n}\right)$ for some $q \in[1, \infty)$. Write $\mathbb{A}_{\infty}\left(\mathbb{R}^{n}\right)=\bigcup_{q \in[1, \infty)} \mathbb{A}_{q}\left(\mathbb{R}^{n}\right)$.

\section{Example 2.4.}

1. Let $\alpha \in \mathbb{R}$ and $1<p<\infty$. It is well known that $|\cdot|^{\alpha} \in A_{p}$ if and only if $-n<\alpha<n(p-1)$ and that $|\cdot|^{\alpha} \in A_{1}$ if and only if $-n<\alpha \leq 0$.

2. By a change of variables and the above facts, we can check that $\min \left(1,|\cdot|^{-n}\right) \notin$ $A_{\infty}$

3. Let $w \in A_{q}$ with $1 \leq q \leq \infty$. If we set $\varphi(x, t) \equiv t^{q} w(x)$, then $\varphi \in \mathbb{A}_{q}\left(\mathbb{R}^{n}\right)$. More generally, if $w \in A_{\infty}$ and $\Phi$ is an Orlicz function, then $\varphi$, given by $\varphi(x, t) \equiv \Phi(t) w(x),(x, t) \in \mathbb{R}^{n} \times(0, \infty)$, belongs to $\mathbb{A}_{\infty}\left(\mathbb{R}^{n}\right)$.

We define Musielak-Orlicz Hardy spaces vie Musielak-Orlicz spaces. We give the standard definition of Musielak-Orlicz spaces.

Definition 2.5. Let $\varphi \in \mathbb{A}_{\infty}\left(\mathbb{R}^{n}\right)$. Then the Musielak-Orlicz space $L^{\varphi}\left(\mathbb{R}^{n}\right)$ is the set of all measurable functions $f: \mathbb{R}^{n} \rightarrow \mathbb{C}$ for which

$$
\int_{\mathbb{R}^{n}} \varphi(x, \varepsilon|f(x)|) d \mathrm{x}<\infty
$$

for some $\varepsilon>0$. For $f \in L^{\varphi}\left(\mathbb{R}^{n}\right)$, the quasi-norm $\|f\|_{L^{\varphi}}$ is given by

$$
\|f\|_{L^{\varphi}} \equiv \inf \left\{\lambda>0: \int_{\mathbb{R}^{n}} \varphi\left(x, \frac{|f(x)|}{\lambda}\right) d \mathrm{x} \leq 1\right\} .
$$

Here is our standing assumption. We suppose that a generalized MusielakOrlicz function $\varphi: \mathbb{R}^{n} \times[0, \infty) \rightarrow[0, \infty)$ satisfies the following conditions:

1. $\varphi \in \mathbb{A}_{\infty}\left(\mathbb{R}^{n}\right)$, so that $q(\varphi)=\inf \left\{q \geq 1: \varphi \in \mathbb{A}_{q}\left(\mathbb{R}^{n}\right)\right\}<\infty$.

2. $\varphi$ is of uniformly lower type $p_{-}$and of uniformly upper type $p_{+}$for some $0<p_{-} \leq p_{+}<\infty$. 
Recall that a growth function is a generalized Musielak-Orlicz function $\varphi \in \mathbb{A}_{\infty}\left(\mathbb{R}^{n}\right)$ of uniformly lower type $p_{-}$and of uniformly upper type $p_{+}=1$, so we tolerate the case where $p_{+}>1$ in this paper.

In the prototype case of $\varphi(x, t)=t^{p}$, we have $q(\varphi)=1, i(\varphi)=I(\varphi)=p$ for any $0<p<\infty$.

It is convenient to note a scaling law:

Lemma 2.6. Let $\varphi \in \mathbb{A}_{\infty}\left(\mathbb{R}^{n}\right)$ and $a>0$. Then

$$
q\left(\varphi\left(\cdot,(\cdot)^{a}\right)\right)=q(\varphi), \quad i\left(\varphi\left(\cdot,(\cdot)^{a}\right)\right)=a i(\varphi), \quad I\left(\varphi\left(\cdot,(\cdot)^{a}\right)\right)=a I(\varphi) .
$$

Proof. We calculate

$$
\left[\varphi\left(\cdot,(\cdot)^{a}\right)\right]_{\mathbb{A}_{q}}=\sup _{t>0}\left[\varphi\left(\cdot, t^{a}\right)\right]_{A_{q}}=\sup _{t>0}[\varphi(\cdot, t)]_{A_{q}}=[\varphi]_{\mathbb{A}_{q}} .
$$

If $p<i(\varphi)$, then $\varphi$ is of uniformly lower type $p$, so that $\varphi(x, s t) \lesssim s^{p} \varphi(x, t)$ for all $0<s \leq 1$ and $t>0$. Thus, $\varphi\left(x, s^{a} t^{a}\right) \lesssim\left(s^{a}\right)^{p} \varphi\left(x, t^{a}\right)$ for all $0<s \leq 1$ and $t>0$, implying that $\varphi\left(\cdot,(\cdot)^{a}\right)$ is of uniformly lower type ap. Thus, ai $(\varphi) \leq i\left(\varphi\left(\cdot,(\cdot)^{a}\right)\right)$. If we swap the role of $\varphi$ and $\varphi\left(\cdot,(\cdot)^{a}\right)$, then we obtain $\operatorname{ai}(\varphi) \geq i\left(\varphi\left(\cdot,(\cdot)^{a}\right)\right)$. Thus, $a i(\varphi)=i\left(\varphi\left(\cdot,(\cdot)^{a}\right)\right)$. If we replace " $0<s \leq 1$ " by " $s \geq 1$ ", then we see $a I(\varphi)=$ $I\left(\varphi\left(\cdot,(\cdot)^{a}\right)\right)$.

What differs crucially from the existing works $[5,17]$ is that we assume only $0<p_{-} \leq p_{+}<\infty$ instead of $0<p_{-} \leq p_{+}=1$.

We will prove an estimate related to the convexity motivated by the paper [17].

Lemma 2.7. Suppose that $\varphi: \mathbb{R}^{n} \times[0, \infty) \rightarrow[0, \infty)$ is a function of uniformly lower type $p$. Then

$$
\varphi\left(x,\left(\sum_{j=1}^{\infty} t_{j}\right)^{\frac{1}{p}}\right) \gtrsim \sum_{j=1}^{\infty} \varphi\left(x, t_{j^{\frac{1}{p}}}\right)
$$

for all non-negative $\ell^{1}$-sequences $\left\{t_{j}\right\}_{j=1}^{\infty}$. In particular, if $p \geq 1$, then

$$
\varphi\left(x, \sum_{j=1}^{\infty} t_{j}\right) \gtrsim \sum_{j=1}^{\infty} \varphi\left(x, t_{j}\right) .
$$

for all non-negative $\ell^{1}$-sequences $\left\{t_{j}\right\}_{j=1}^{\infty}$. If $\varphi: \mathbb{R}^{n} \times[0, \infty) \rightarrow[0, \infty)$ is a function strictly of uniformly lower type $p$, then $\gtrsim$ can be replaced by $\geq$.

Proof. Write $T=\sum_{j=1}^{\infty} t_{j}$. Since the mapping $(x, t) \mapsto \varphi\left(x, t^{\frac{1}{p}}\right)$ is of uniformly lower type 1 ,

$$
\frac{t_{k}}{T} \varphi\left(x, T^{\frac{1}{p}}\right) \gtrsim \varphi\left(x, t_{k}^{\frac{1}{p}}\right)
$$

for all $k \in \mathbb{N}$. It remains to add this estimate over $k$.

The following observation is crucial for later considerations: 
Lemma 2.8. Let $0<p_{-} \leq p_{+}<\infty$. Let $\varphi: \mathbb{R}^{n} \times[0, \infty) \rightarrow[0, \infty)$ be a generalized Musielak-Orlicz function of uniformly lower type $p_{-}$and of uniformly upper type $p_{+}$. Then there exists a generalized Musielak-Orlicz function $\tilde{\varphi}: \mathbb{R}^{n} \times[0, \infty) \rightarrow$ $[0, \infty)$ strictly of uniformly lower type $p_{-}$and strictly of uniformly upper type $p_{+}$ such that $\varphi(x, t) \sim \tilde{\varphi}(x, t)$ for all $x, t$.

Proof. First of all, we set $\psi(x, t) \equiv \sup _{s \in(0,1]} s^{-p_{-}} \varphi(x, s t)$ for $(x, t) \in \mathbb{R}^{n} \times[0, \infty)$. Then since $\varphi$ is of uniformly lower type $p_{-}, \varphi(x, t) \sim \psi(x, t)$ for all $x, t$, so that $\psi$ is of uniformly upper type $p_{+}$. Furthermore, for $0<v \leq 1, x \in \mathbb{R}^{n}$ and $t>0$, arithmetic shows

$$
\begin{aligned}
\psi(x, v t) & =\sup _{s \in(0,1]} s^{-p_{-}} \varphi(x, s v t) \\
& =v^{p_{-}} \sup _{s \in(0,1]}(s v)^{-p_{-}} \varphi(x, s v t) \\
& =v^{p_{-}} \sup _{s \in(0, v]} s^{-p_{-}} \varphi(x, s t) .
\end{aligned}
$$

By enlarging the range of $v$, we obtain

$$
\psi(x, v t) \leq v^{p_{-}} \sup _{s \in(0,1]} s^{-p_{-}} \varphi(x, s t)=v^{p_{-}} \psi(x, t) .
$$

Thus, $\psi$ is strictly of uniformly lower type $p_{-}$.

Next, we define $\tilde{\varphi}(x, t)=\sup _{s \geq 1} s^{-p_{+}} \psi(x, s t)$ for $(x, t) \in \mathbb{R}_{+}^{n+1}$. Then since $\psi$ is of uniformly upper type $p_{+}, \varphi(x, t) \sim \tilde{\varphi}(x, t) \sim \psi(x, t)$ for all $(x, t) \in \mathbb{R}_{+}^{n+1}$. For $v \in(0,1]$, we estimate

$$
\tilde{\varphi}(x, v t)=\sup _{s \geq 1} s^{-p_{+}} \psi(x, v s t) \leq v^{p_{-}} \sup _{s \geq 1} s^{-p_{+}} \psi(x, s t) \leq v^{p_{-}} \tilde{\varphi}(x, t)
$$

since $\psi$ is strictly of uniformly lower type $p_{-}$. Thus, $\tilde{\varphi}$ is strictly of uniformly lower type $p_{-}$again. Meanwhile, for $v \geq 1$, arithmetic shows that

$$
\tilde{\varphi}(x, v t)=\sup _{s \geq 1} s^{-p_{+}} \psi(x, s v t)=v^{p_{+}} \sup _{s \geq v} s^{-p_{+}} \psi(x, s t)
$$

and hence

$$
\tilde{\varphi}(x, v t) \leq v^{p_{+}} \sup _{s \geq 1} s^{-p} \psi(x, s t)=v^{p_{+}} \tilde{\varphi}(x, v) .
$$

Consequently, we obtain the desired function $\tilde{\varphi}$.

There are several works dealing with Musielak-Orlicz spaces under various settings. Here we compare ones in this paper and ones in other papers.

\section{Remark 2.9.}


1. As in [15], the class $\Phi$ of generalized Young functions is the set of all measurable functions $\Phi:[0, \infty] \rightarrow[0, \infty]$ such that $\Phi(0)=\lim _{x \rightarrow 0} \Phi(x)=0$ and $\lim _{x \rightarrow \infty} \Phi(x)=\infty$.

2. Let $\Phi \in \Phi$ and $\ell \in(0, \infty)$. One says that $\Phi$ is $\ell$-convex/concave if $\Phi\left((\cdot)^{1 / \ell}\right)$ is convex/concave.

3. The class $\Phi_{0}$ stands for the set of all $\Phi \in \Phi$ which is equivalent to an $\ell_{-}$-convex generalized Young function $\Phi_{-}$and to an $\ell_{+}$-concave generalized Young function $\Phi_{+}$for some $0<\ell_{-} \leq \ell_{+}<\infty$.

4. Let $0<\ell_{-} \leq \ell_{+}<\infty$. Then the function $\varphi: \mathbb{R}^{n} \times[0, \infty) \rightarrow[0, \infty)$, given by

$$
\varphi(x, t)=\Phi(t) \quad\left(x, t \in \mathbb{R}^{n} \times[0, \infty)\right),
$$

belongs to $\mathbb{A}_{1}\left(\mathbb{R}^{n}\right)$ for any $\Phi \in \Phi$ which is equivalent to an $\ell_{-}$-convex generalized Young function $\Phi_{-}$and to an $\ell_{+}$-concave generalized Young function $\Phi_{+}$.

5. In [22], the second and the third authors investigated Orlicz-Hardy spaces. In view of the above observation, it follows that Musielak-Orlicz Hardy spaces considered in this paper will cover Orlicz-Hardy spaces in [22]. In [22, Section 8], the weighted setting is considered. If $\varphi$ is given by $\varphi(x, t)=\Phi(t) w(x)$ for some $\Phi \in \Phi$ and $w \in A_{\infty}$, then $\varphi \in \mathbb{A}_{\infty}\left(\mathbb{R}^{n}\right)$ and $H^{\varphi}\left(\mathbb{R}^{n}\right)$, whose definition is given in Subsection 3.1, coincides with the weighted Orlicz-Hardy space $H^{\Phi}(w)$ considered in [22, Section 8].

\section{Remark 2.10.}

1. In [21, Example 2.2], Maeda, the third author and Shimomura considered a function

$$
\varphi(x, t)=t \phi(x, t): \mathbb{R}^{n} \times[0, \infty) \rightarrow[0, \infty) .
$$

Consider $(\varphi 1)-(\varphi 3)$ below, which correspond to $(\Phi 1)-(\Phi 3)$ in $[21]$, respectively.

$(\varphi 1) \phi(\cdot, t)$ is measurable on $\mathbb{R}^{n}$ for each $t \geq 0$ and $\phi(x, \cdot)$ is continuous on $[0, \infty)$ for each $x \in \mathbb{R}^{n}$;

$(\varphi 2)$ there exists a constant $A_{1} \geq 1$ such that $A_{1}^{-1} \leq \phi(x, 1) \leq A_{1}$ for all $x \in \mathbb{R}^{n}$, so that $\log \phi(\cdot, 1) \in L^{\infty}\left(\mathbb{R}^{n}\right) ;$

$(\varphi 3) \phi(x, \cdot)$ is uniformly almost increasing on $(0, \infty)$, namely there exists a constant $A_{2} \geq 1$ such that $\phi(x, t) \leq A_{2} \phi(x, a t)$ for all $x \in \mathbb{R}^{n}, t>0$ and $a>1$.

Condition $(\varphi 3)$ implies that $\varphi$ is of lower type 1 .

2. Let $\bar{\phi}(x, t)=\sup _{0 \leq s \leq t} \phi(x, s)$ and

$$
\bar{\varphi}(x, t)=\int_{0}^{t} \bar{\phi}(x, r) d r \quad\left((x, t) \in \mathbb{R}^{n} \times[0, \infty)\right)
$$


for $x \in \mathbb{R}^{n}$ and $t \geq 0$. Then $\bar{\phi}(x, \cdot)$ is continuous nondecreasing, $\bar{\varphi}(x, \cdot)$ is convex and

$$
\varphi(x, t / 2) \leq \bar{\varphi}(x, t) \leq A_{2} \varphi(x, t)
$$

for all $x \in \mathbb{R}^{n}$ and $t \geq 0$.

3. We will also consider the following conditions on $\phi$ and $\varphi$ satisfying (2.3): Let $\varepsilon \geq 0, \nu>0$ and $\omega>0$.

$(\varphi 3 ; \varepsilon) \quad t \mapsto t^{-\varepsilon} \phi(x, t)$ is uniformly almost increasing on $(0, \infty)$, namely there exists a constant $A_{2, \varepsilon} \geq 1$ such that

$$
\phi(x, t) \leq A_{2, \varepsilon} a^{-\varepsilon} \phi(x, a t) \quad \text { for all } x \in \mathbb{R}^{n} \text { whenever } t>0 \text { and } a>1 ;
$$

$(\varphi 4) \phi(x, \cdot)$ satisfies the uniform doubling condition, namely there exists a constant $A_{3} \geq 1$ such that

$$
\phi(x, 2 t) \leq A_{3} \phi(x, t) \quad \text { for all } x \in \mathbb{R}^{n} \text { and } t>0 ;
$$

$(\varphi 5 ; \nu)$ For every $\gamma>0$, there exists a constant $B_{\gamma, \nu} \geq 1$ such that

$$
\varphi(x, t) \leq B_{\gamma, \nu} \varphi(y, t)
$$

whenever $|x-y| \leq \gamma t^{-\nu}$ and $t \geq 1$;

$(\varphi 6 ; \omega)$ there exist a function $g$ on $\mathbb{R}^{n}$ and a constant $B_{\infty} \geq 1$ such that $0 \leq g(x)<1$ for all $x \in \mathbb{R}^{n}, g^{\omega} \in L^{1}\left(\mathbb{R}^{n}\right)$ and

$$
B_{\infty}^{-1} \varphi(x, t) \leq \varphi\left(x^{\prime}, t\right) \leq B_{\infty} \varphi(x, t)
$$

whenever $\left|x^{\prime}\right| \geq|x|$ and $g(x) \leq t \leq 1$.

Under condition $(\varphi 4), \varphi$ is of upper type $p_{+}$for some $p_{+} \in[1, \infty)$.

4. Remark that there exists a function $\varphi \in \mathbb{A}_{\infty}\left(\mathbb{R}^{n}\right)$ such that condition $(\varphi 2)$ fails.

5. For $p \geq 1, q>0$ and $r>0$, set

$$
\varphi(x, t)= \begin{cases}t^{p} \max \left(1, t^{q} \min (1,|x|)\right) & \text { if } t \geq 1, \\ t^{p} \max \left(t, \min \left(1 / 2,|x|^{-N / r}\right)\right) & \text { if } t<1 .\end{cases}
$$

We collect some known facts from [21]. First, $\varphi(x, t)$ satisfies $(\varphi j), j=$ $1,2,3,4$; it satisfies $(\varphi 3 ; p-1)$.

(a) $\varphi(x, t)$ satisfies $(\varphi 5 ; \nu)$ if and only if $\nu \geq q$;

(b) $\varphi(x, t)$ satisfies $(\varphi 6 ; \omega)$ if $\omega>r$ but does not satisfy $(\varphi 6 ; \omega)$ if $\omega<r$.

Let $r=1$ here and below. Then since $\min \left(1,|\cdot|^{-N}\right) \notin A_{\infty}\left(\mathbb{R}^{n}\right)$ according to Example 2.4, it follows that $\varphi \notin \mathbb{A}_{\infty}\left(\mathbb{R}^{n}\right)$.

It is noteworthy that Musielak-Orlicz spaces considered in [21] are designed to extend Lebesgue spaces with variable exponents. 


\subsection{Boundedness of the Hardy-Littlewood maximal oper- ator}

The goal of Subsection 2.2 is to investigate the boundedness property of the HardyLittlewood maximal operator, whose definition we recall now. We write $Q(x, r)$ for a cube which has center $x$ and radius $r$, that is,

$$
Q(x, r) \equiv\left\{y=\left(y_{1}, y_{2}, \ldots, y_{n}\right) \in \mathbb{R}^{n}: \max _{1 \leq j \leq n}\left|x_{j}-y_{j}\right| \leq r\right\}
$$

when $x=\left(x_{1}, x_{2}, \ldots, x_{n}\right) \in \mathbb{R}^{n}$ and $r>0$. Let $0<\eta<\infty$. We define the powered Hardy-Littlewood maximal operator $M^{(\eta)}$ by

$$
M^{(\eta)} f(x) \equiv \sup _{R>0}\left(\frac{1}{|Q(x, R)|} \int_{Q(x, R)}|f(y)|^{\eta} \mathrm{d} y\right)^{\frac{1}{\eta}} .
$$

We simply write $M=M^{(1)}$.

We will invoke the following vector-valued boundedness of the Hardy-Littlewood maximal operator.

Lemma 2.11. [29, Theorem 2.1.4] Let $1<r \leq \infty$. Assume that $\varphi \in \mathbb{A}_{\infty}\left(\mathbb{R}^{n}\right)$. If $q(\varphi)<p_{-} \leq p_{+}<\infty$, then there exists a constant $C>0$ such that

$$
\int_{\mathbb{R}^{n}} \varphi\left(x,\left\{\sum_{j=-\infty}^{\infty} M f_{j}(x)^{r}\right\}^{\frac{1}{r}}\right) d \mathrm{x} \leq C \int_{\mathbb{R}^{n}} \varphi\left(x,\left\{\sum_{j=-\infty}^{\infty}\left|f_{j}(x)\right|^{r}\right\}^{\frac{1}{r}}\right) d \mathrm{x}
$$

for all sequences of measurable functions $\left\{f_{j}\right\}_{j=-\infty}^{\infty}$. In particular,

$$
\left\|\left(\sum_{j=-\infty}^{\infty} M f_{j}^{r}\right)^{\frac{1}{r}}\right\|_{L^{\varphi}} \leq C\left\|\left(\sum_{j=-\infty}^{\infty}\left|f_{j}\right|^{r}\right)^{\frac{1}{r}}\right\|_{L^{\varphi}} .
$$

Here a natural modification is made when $r=\infty$.

We let

$$
d_{\varphi} \equiv \max \left\{\left[n\left(\frac{q(\varphi)}{i(\varphi)}-1\right)\right], 0\right\} .
$$

A direct consequence of Lemma 2.11 is that

$$
\left\|\chi_{Q}\right\|_{L^{\varphi}} \simeq\left\|\chi_{2 \sqrt{n} Q}\right\|_{L^{\varphi}}
$$

for all cubes $Q$, since $\chi_{Q} \leq \chi_{2 \sqrt{n} Q} \lesssim\left(M \chi_{Q}\right)^{r}$ for any $r>0$.

As we have been mentioning, we are interested in $\varphi \in \mathbb{A}_{\infty}\left(\mathbb{R}^{n}\right)$ satisfying $0<$ $i(\varphi) \leq I(\varphi)<\infty$. Thus, the postulate $q(\varphi)<i(\varphi)$ in Lemma 2.11 is too strong. To overcome this problem, we transform Lemma 2.11 into the following form:

Corollary 2.12. For $\varphi \in \mathbb{A}_{\infty}\left(\mathbb{R}^{n}\right)$ satisfying $0<i(\varphi) \leq I(\varphi)<\infty$ and for all sequences of measurable functions $\left\{f_{j}\right\}_{j=-\infty}^{\infty}$,

$$
\int_{\mathbb{R}^{n}} \varphi\left(x, \sum_{j=-\infty}^{\infty} M f_{j}(x)^{\frac{n+d \varphi+1}{n}}\right) d \mathrm{x} \lesssim \int_{\mathbb{R}^{n}} \varphi\left(x, \sum_{j=-\infty}^{\infty}\left|f_{j}(x)\right|^{\frac{n+d_{\varphi}+1}{n}}\right) d \mathrm{x} .
$$


Proof. We consider $\varphi\left(\cdot,(\cdot)^{\frac{n+d_{\varphi}+1}{n}}\right)$. We calculate

$$
q\left(\varphi\left(\cdot,(\cdot)^{\frac{n+d_{\varphi}+1}{n}}\right)\right)=q(\varphi), \quad i\left(\varphi\left(\cdot,(\cdot)^{\frac{n+d_{\varphi}+1}{n}}\right)\right)=\frac{n+d_{\varphi}+1}{n} i(\varphi)>q(\varphi)
$$

using Lemma 2.6, since $d_{\varphi}+1>n\left(\frac{q(\varphi)}{i(\varphi)}-1\right)$. Thus, we are in the position of using Lemma 2.11 to control the Hardy-Littlewood maximal operator in the right-hand side of (2.8).

Definition 2.13. Let $\varphi \in \mathbb{A}_{\infty}\left(\mathbb{R}^{n}\right)$.

1. Let $\underline{\varphi} \equiv \min \{1, i(\varphi)\} \in(0,1]$, where $i(\varphi)$ is given by $(2.1)$.

2. For sequences of nonnegative numbers $\left\{\kappa_{j}\right\}_{j=1}^{\infty}$ and cubes $\left\{Q_{j}\right\}_{j=1}^{\infty}$, define

$$
\begin{aligned}
& \mathcal{A}\left(\left\{\kappa_{j}\right\}_{j=1}^{\infty},\left\{Q_{j}\right\}_{j=1}^{\infty}\right)=\mathcal{A}_{\varphi}\left(\left\{\kappa_{j}\right\}_{j=1}^{\infty},\left\{Q_{j}\right\}_{j=1}^{\infty}\right) \\
& \equiv \inf \left\{\lambda>0: \int_{\mathbb{R}^{n}} \varphi\left(x,\left\{\sum_{j=1}^{\infty}\left(\frac{\kappa_{j} \chi_{Q_{j}}(x)}{\lambda\left\|\chi_{Q_{j}}\right\|_{L^{\varphi}}}\right)^{\underline{\varphi}}\right\}^{1 / \underline{\varphi}}\right) d \mathrm{x} \leq 1\right\} .
\end{aligned}
$$

If $\varphi(x, t)=t^{p}$, then write $\mathcal{A}_{\varphi}\left(\left\{\kappa_{j}\right\}_{j=1}^{\infty},\left\{Q_{j}\right\}_{j=1}^{\infty}\right)=\mathcal{A}_{p}\left(\left\{\kappa_{j}\right\}_{j=1}^{\infty},\left\{Q_{j}\right\}_{j=1}^{\infty}\right)$.

3. If $E_{j}$ is a measurable subset of $Q_{j}$ for each $j \in \mathbb{N}$, define

$$
\begin{aligned}
& \mathcal{A}\left(\left\{\kappa_{j}\right\}_{j=1}^{\infty},\left\{E_{j}\right\}_{j=1}^{\infty},\left\{Q_{j}\right\}_{j=1}^{\infty}\right)=\mathcal{A}_{\varphi}\left(\left\{\kappa_{j}\right\}_{j=1}^{\infty},\left\{E_{j}\right\}_{j=1}^{\infty},\left\{Q_{j}\right\}_{j=1}^{\infty}\right) \\
& \equiv \inf \left\{\lambda>0: \int_{\mathbb{R}^{n}} \varphi\left(x,\left\{\sum_{j=1}^{\infty}\left(\frac{\kappa_{j} \chi_{E_{j}}(x)}{\lambda\left\|\chi_{Q_{j}}\right\|_{L^{\varphi}}}\right)^{\underline{\varphi}}\right\}^{1 / \underline{\varphi}}\right) d \mathrm{x} \leq 1\right\} .
\end{aligned}
$$

If $\varphi(x, t)=t^{p}$, then write

$$
\mathcal{A}_{\varphi}\left(\left\{\kappa_{j}\right\}_{j=1}^{\infty},\left\{E_{j}\right\}_{j=1}^{\infty},\left\{Q_{j}\right\}_{j=1}^{\infty}\right)=\mathcal{A}_{p}\left(\left\{\kappa_{j}\right\}_{j=1}^{\infty},\left\{E_{j}\right\}_{j=1}^{\infty},\left\{Q_{j}\right\}_{j=1}^{\infty}\right) .
$$

By the use of the Hardy-Littlewood maximal operator, we have the following: Proposition 2.14. Assume that $\varphi \in \mathbb{A}_{\infty}\left(\mathbb{R}^{n}\right)$ satisfies $0<i(\varphi) \leq I(\varphi)<\infty$. For each $j \in \mathbb{N}$, suppose that we have $E_{j}$ is a measurable subset of $Q_{j}$ satisfying $2\left|E_{j}\right| \geq\left|Q_{j}\right|$. Then, for all $\left\{\kappa_{j}\right\}_{j=1}^{\infty} \subset[0, \infty)$,

$$
\mathcal{A}\left(\left\{\kappa_{j}\right\}_{j=1}^{\infty},\left\{Q_{j}\right\}_{j=1}^{\infty}\right) \lesssim \mathcal{A}\left(\left\{\kappa_{j}\right\}_{j=1}^{\infty},\left\{E_{j}\right\}_{j=1}^{\infty},\left\{Q_{j}\right\}_{j=1}^{\infty}\right) .
$$

Proof. By the normalization, we may assume $\mathcal{A}\left(\left\{\kappa_{j}\right\}_{j=1}^{\infty},\left\{E_{j}\right\}_{j=1}^{\infty},\left\{Q_{j}\right\}_{j=1}^{\infty}\right)=1$. Remark that $M$ is given by (2.5). Since $\chi_{Q_{j}} \leq c_{n} M \chi_{E_{j}}$ for some $c_{n} \geq 1$, we obtain, for $a \gg 1$,

$$
\begin{aligned}
& \int_{\mathbb{R}^{n}} \varphi\left(x,\left\{\sum_{j=1}^{\infty}\left(\frac{\kappa_{j} \chi_{Q_{j}}(x)}{\left\|\chi_{Q_{j}}\right\|_{L^{\varphi}}}\right)^{\underline{\varphi}}\right\}^{1 / \underline{\varphi}}\right) d \mathrm{x} \\
& \leq \int_{\mathbb{R}^{n}} \varphi\left(x,\left\{\sum_{j=1}^{\infty}\left(\frac{c_{n}{ }^{a} \kappa_{j} M \chi_{E_{j}}(x)^{a}}{\left\|\chi_{Q_{j}}\right\|_{L^{\varphi}}}\right)^{\underline{\varphi}}\right\}^{1 / \underline{\varphi}}\right) d \mathrm{x} \\
& =\int_{\mathbb{R}^{n}} \varphi\left(x,\left\{\sum_{j=1}^{\infty}\left(\frac{c_{n}\left(\kappa_{j}\right)^{1 / a} M \chi_{E_{j}}(x)}{\left(\left\|\chi_{Q_{j}}\right\|_{L^{\varphi}}\right)^{1 / a}}\right)^{a \underline{\varphi}}\right\}^{1 /(a \underline{\varphi})}\right) d \mathrm{x} .
\end{aligned}
$$


Thanks to Lemma 2.6, $q\left(\varphi\left(\cdot,(\cdot)^{a}\right)\right)<i\left(\varphi\left(\cdot,(\cdot)^{a}\right)\right) \leq I\left(\varphi\left(\cdot,(\cdot)^{a}\right)\right)<\infty$ as long as $a \gg 1$. Thus, we are in the position of using Lemma 2.11 to have:

$$
\begin{aligned}
& \int_{\mathbb{R}^{n}} \varphi\left(x,\left\{\sum_{j=1}^{\infty}\left(\frac{\kappa_{j} \chi_{Q_{j}}(x)}{\left\|\chi_{Q_{j}}\right\|_{L^{\varphi}}}\right)^{\underline{\varphi}}\right\}^{1 / \underline{\varphi}}\right) d \mathrm{x} \\
& \lesssim \int_{\mathbb{R}^{n}} \varphi\left(x,\left\{\sum_{j=1}^{\infty}\left(\frac{\kappa_{j} \chi_{E_{j}}(x)}{\left\|\chi_{Q_{j}}\right\|_{L^{\varphi}}}\right)^{\underline{\varphi}}\right\}^{1 / \underline{\varphi}}\right) d \mathrm{x}=1 .
\end{aligned}
$$

Since $i(\varphi)>0$, we conclude

$$
\int_{\mathbb{R}^{n}} \varphi\left(x,\left\{\sum_{j=1}^{\infty}\left(\frac{\kappa_{j} \chi_{Q_{j}}(x)}{C\left\|\chi_{Q_{j}}\right\|_{L^{\varphi}}}\right)^{\underline{\varphi}}\right\}^{1 / \underline{\varphi}}\right) d \mathrm{x} \leq 1
$$

for some constant $C>0$. Thus, the proof is complete.

We will assume that $\varphi\left(\cdot,(\cdot)^{1 / \underline{\varphi}}\right)$ is strictly of uniformly lower type 1 and strictly of uniformly upper type $P \geq 1$ thanks to Lemma 2.8 .

Proposition 2.15. Assume that $\varphi \in \mathbb{A}_{\infty}\left(\mathbb{R}^{n}\right)$ satisfies $0<i(\varphi) \leq I(\varphi)<\infty$. Assume that $\varphi\left(\cdot,(\cdot)^{1 / \underline{\varphi}}\right)$ is strictly of uniformly lower type 1 and strictly of uniformly upper type $P \geq 1$. For each $j \in \mathbb{N}$, suppose that we have $E_{j}$ is a measurable subset of $Q_{j}$ satisfying $2\left|E_{j}\right| \leq\left|Q_{j}\right|$. Then there exists $\beta \in(0,1)$ such that for all $\left\{\kappa_{j}\right\}_{j=1}^{\infty} \subset[0, \infty), \mathcal{A}\left(\left\{\kappa_{j}\right\}_{j=1}^{\infty},\left\{E_{j}\right\}_{j=1}^{\infty},\left\{Q_{j}\right\}_{j=1}^{\infty}\right) \leq \beta \mathcal{A}\left(\left\{\kappa_{j}\right\}_{j=1}^{\infty},\left\{Q_{j}\right\}_{j=1}^{\infty}\right)$.

Proof. We may assume that $\mathcal{A}\left(\left\{\kappa_{j}\right\}_{j=1}^{\infty},\left\{Q_{j}\right\}_{j=1}^{\infty}\right)=1$ by normalization. Since $\varphi$ is strictly of lower type $1, \varphi(x, a) \leq \frac{a}{a+b} \varphi(x, a+b)$ for all $a, b>0$ and $x \in \mathbb{R}^{n}$ and hence

$$
\varphi(x, a)+\varphi(x, b) \leq \varphi(x, a+b)
$$

for all $a, b>0$ and $x \in \mathbb{R}^{n}$. Since

$$
\sum_{j=1}^{\infty}\left(\frac{\kappa_{j} \chi_{Q_{j}}}{\left\|\chi_{Q_{j}}\right\|_{L^{\varphi}}}\right)^{\underline{\varphi}}=\sum_{j=1}^{\infty}\left(\frac{\kappa_{j} \chi_{E_{j}}}{\left\|\chi_{Q_{j}}\right\|_{L^{\varphi}}}\right)^{\underline{\varphi}}+\sum_{j=1}^{\infty}\left(\frac{\kappa_{j} \chi_{Q_{j} \backslash E_{j}}}{\left\|\chi_{Q_{j}}\right\|_{L^{\varphi}}}\right)^{\underline{\varphi}}
$$

we have

$$
\begin{aligned}
& \varphi\left(x,\left\{\sum_{j=1}^{\infty}\left(\frac{\kappa_{j} \chi_{Q_{j}}(x)}{\left\|\chi_{Q_{j}}\right\|_{L^{\varphi}}}\right)^{\underline{\varphi}}\right\}^{1 / \underline{\varphi}}\right) \\
& \geq \varphi\left(x,\left\{\sum_{j=1}^{\infty}\left(\frac{\kappa_{j} \chi_{E_{j}}(x)}{\left\|\chi_{Q_{j}}\right\|_{L^{\varphi}}}\right)^{\underline{\varphi}}\right\}^{1 / \underline{\varphi}}\right)+\varphi\left(x,\left\{\sum_{j=1}^{\infty}\left(\frac{\kappa_{j} \chi_{Q_{j} \backslash E_{j}}(x)}{\left\|\chi_{Q_{j}}\right\|_{L^{\varphi}}}\right)^{\underline{\varphi}}\right\}^{1 / \underline{\varphi}}\right)
\end{aligned}
$$

thanks to (2.11). Thus, integrating this esimate over $\mathbb{R}^{n}$, we obtain

$$
\begin{aligned}
1 \geq & \int_{\mathbb{R}^{n}} \varphi\left(x,\left\{\sum_{j=1}^{\infty}\left(\frac{\kappa_{j} \chi_{E_{j}}(x)}{\left\|\chi_{Q_{j}}\right\|_{L^{\varphi}}}\right)^{\underline{\varphi}}\right\}^{1 / \underline{\varphi}}\right) d \mathrm{x} \\
& +\int_{\mathbb{R}^{n}} \varphi\left(x,\left\{\sum_{j=1}^{\infty}\left(\frac{\kappa_{j} \chi_{Q_{j} \backslash E_{j}}(x)}{\left\|\chi_{Q_{j}}\right\|_{L^{\varphi}}}\right)^{\underline{\varphi}}\right\}^{1 / \underline{\varphi}}\right) d \mathrm{x} .
\end{aligned}
$$


We know that

$$
\int_{\mathbb{R}^{n}} \varphi\left(x,\left\{D \sum_{j=1}^{\infty}\left(\frac{\kappa_{j} \chi_{Q_{j} \backslash E_{j}}(x)}{\left\|\chi_{Q_{j}}\right\|_{L^{\varphi}}}\right)^{\underline{\varphi}}\right\}^{1 / \underline{\varphi}}\right) d \mathrm{x} \geq 1
$$

for some $D>1$ thanks to Proposition 2.14. Since $\varphi\left(\cdot,(\cdot)^{1 / \underline{\varphi}}\right)$ is assumed to be of lower type 1 , we have

$$
\int_{\mathbb{R}^{n}} \varphi\left(x,\left\{\sum_{j=1}^{\infty}\left(\frac{\kappa_{j} \chi_{Q_{j} \backslash E_{j}}(x)}{\left\|\chi_{Q_{j}}\right\|_{L^{\varphi}}}\right)^{\underline{\varphi}}\right\}^{1 / \underline{\varphi}}\right) d \mathrm{x} \geq D^{-1} .
$$

Thus, letting $\beta=\left(1-D^{-1}\right)^{\frac{1}{P}}$, we obtain

$$
\int_{\mathbb{R}^{n}} \varphi\left(x,\left\{\sum_{j=1}^{\infty}\left(\frac{\kappa_{j} \chi_{E_{j}}(x)}{\left\|\chi_{Q_{j}}\right\|_{L^{\varphi}}}\right)^{\underline{\varphi}}\right\}^{1 / \underline{\varphi}}\right) d \mathrm{x} \leq \beta^{P} .
$$

Since $\varphi\left(\cdot,(\cdot)^{1 / \underline{\varphi}}\right)$ is of uniformly upper type $P \geq 1$, we obtain

$$
\int_{\mathbb{R}^{n}} \varphi\left(x,\left\{\beta^{-1} \sum_{j=1}^{\infty}\left(\frac{\kappa_{j} \chi_{E_{j}}(x)}{\left\|\chi_{Q_{j}}\right\|_{L^{\varphi}}}\right)^{\underline{\varphi}}\right\}^{1 / \underline{\varphi}}\right) d \mathrm{x} \leq 1
$$

as required.

Given a cube $Q$, let us denote by $L^{1}(Q)$ the set of all integrable functions supported on $Q$.

Lemma 2.16. Let $\varphi \in \mathbb{A}_{\infty}\left(\mathbb{R}^{n}\right)$ satisfy $0<i(\varphi) \leq I(\varphi)<\infty$. Let $\beta$ be a constant from Proposition 2.15 and

$$
\delta \in\left(0, \frac{-\log _{2} \beta}{n+1}\right) .
$$

Suppose that we are given a countable collection of nonnegative numbers $\left\{\kappa_{j}\right\}_{j=1}^{\infty}$, cubes $\left\{Q_{j}\right\}_{j=1}^{\infty}$ and non-zero measurable functions $\left\{f_{j}\right\}_{j=1}^{\infty}$ such that $f_{j} \in L^{1}\left(Q_{j}\right)$ for each $j \in \mathbb{N}$. Then we have

$$
\left\|\left\{\sum_{j=1}^{\infty}\left(\frac{\kappa_{j}\left|f_{j}\right|^{\delta}\left|Q_{j}\right|^{\delta}}{\left\|f_{j}\right\|_{L^{1}\left(Q_{j}\right)}^{\delta}\left\|\chi_{Q_{j}}\right\|_{L^{\varphi}}}\right)^{\underline{\varphi}}\right\}^{\frac{1}{\varphi}}\right\|_{L^{\varphi}} \lesssim \mathcal{A}\left(\left\{\kappa_{j}\right\}_{j=1}^{\infty},\left\{Q_{j}\right\}_{j=1}^{\infty}\right) .
$$

Proof. Fix $j \in \mathbb{N}$. Denote by $M^{d, Q_{j}}$ the dyadic maximal operator with respect to $Q_{j}$. Let us set

$$
E_{j, 0} \equiv Q_{j}, \quad E_{j, k} \equiv\left\{x \in Q_{j}: \frac{2^{(n+1)(k-1)}}{\left|Q_{j}\right|} \int_{Q_{j}}\left|f_{j}(y)\right| d \mathrm{y}<M^{d, Q_{j}} f_{j}(x)\right\}, \quad k \in \mathbb{N} .
$$


Then each $E_{j, k}$ can be partitioned into disjoint maximal dyadic cubes $\left\{Q_{j, k, l}\right\}_{l \in L_{j, k}}$ such that

$$
E_{j, k}=\bigcup_{l \in L_{j, k}} Q_{j, k, l}, \quad \frac{2^{(n+1)(k-1)}}{\left|Q_{j}\right|} \int_{Q_{j}}\left|f_{j}(y)\right| d \mathrm{y}<\frac{1}{\left|Q_{j, k, l}\right|} \int_{Q_{j, k, l}}\left|f_{j}(y)\right| d \mathrm{y} .
$$

Fix $j \in \mathbb{N}$. In the course of the proof of [23, Lemma 4.10], we established that $\left\{Q_{j, k, l}\right\}_{k \in \mathbb{Z}, l \in L_{j, k}}$ satisfies $2\left|Q_{j, k, l} \cap E_{j, k+1}\right| \leq\left|Q_{j, k, l}\right|$ for each $l \in L_{j, k}$.

We decompose

$$
\begin{aligned}
\left\|\left\{\sum_{j=1}^{\infty}\left(\frac{\kappa_{j} \chi_{E_{j, k+1}}}{\left\|\chi_{Q_{j}}\right\|_{L^{\varphi}}}\right)^{\underline{\varphi}}\right\}^{\frac{1}{\varphi}}\right\|_{L^{\varphi}} & =\left\|\left\{\sum_{j=1}^{\infty}\left(\sum_{l \in L_{j, k}} \frac{\kappa_{j} \chi_{Q_{j, k, l} \cap E_{j, k+1}}}{\left\|\chi_{Q_{j}}\right\|_{L^{\varphi}}}\right)^{\underline{\varphi}}\right\}^{\frac{1}{\varphi}}\right\|_{L^{\varphi}} \\
& =\left\|\left\{\sum_{j=1}^{\infty} \sum_{l \in L_{j, k}}\left(\frac{\kappa_{j} \chi_{Q_{j, k, l} \cap E_{j, k+1}}}{\left\|\chi_{Q_{j}}\right\|_{L^{\varphi}}}\right)^{\underline{\varphi}}\right\}^{\underline{\varphi}}\right\|_{L^{\varphi}}
\end{aligned}
$$

by virtue of the fact that $\left\{Q_{j, k, l}\right\}_{l \in L_{j, k}}$ are disjoint. If we invoke Proposition 2.15, then we have

$$
\begin{aligned}
\left\|\left\{\sum_{j=1}^{\infty}\left(\frac{\kappa_{j} \chi_{Q_{j, k, l} \cap E_{j, k+1}}}{\left\|\chi_{Q_{j}}\right\|_{L^{\varphi}}}\right)^{\underline{\varphi}}\right\}^{\frac{1}{\varphi}}\right\|_{L^{\varphi}} & \leq \beta\left\|\left\{\sum_{j=1}^{\infty} \sum_{l \in L_{j, k}}\left(\frac{\kappa_{j} \chi_{Q_{j, k, l}}}{\left\|\chi_{Q_{j}}\right\|_{L^{\varphi}}}\right)^{\underline{\varphi}}\right\}^{\frac{1}{\varphi}}\right\|_{L^{\varphi}} \\
& =\beta\left\|\left\{\sum_{j=1}^{\infty}\left(\frac{\kappa_{j} \chi_{E_{j, k}}}{\left\|\chi_{Q_{j}}\right\|_{L^{\varphi}}}\right)^{\underline{\varphi}}\right\}^{\frac{1}{\varphi}}\right\|_{L^{\varphi}} .
\end{aligned}
$$

Hence it follows that

$$
\left\|\left\{\sum_{j=1}^{\infty}\left(\frac{\kappa_{j} \chi_{E_{j, k+1}}}{\left\|\chi_{Q_{j}}\right\|_{L^{\varphi}}}\right)^{\underline{\varphi}}\right\}^{\frac{1}{\varphi}}\right\|_{L^{\varphi}} \leq \beta\left\|\left\{\sum_{j=1}^{\infty}\left(\frac{\kappa_{j} \chi_{E_{j, k}}}{\left\|\chi_{Q_{j}}\right\|_{L^{\varphi}}}\right)^{\underline{\varphi}}\right\}^{\frac{1}{\varphi}}\right\|_{L^{\varphi}}
$$

for all $k \in \mathbb{N} \cup\{0\}$. If we repeat to use (2.13), then we have

$$
\left.\|\left\{\sum_{j=1}^{\infty}\left(\frac{\kappa_{j} \chi_{E_{j, k}}}{\left\|\chi_{Q_{j}}\right\|_{L^{\varphi}}}\right)^{\underline{\varphi}}\right)\right\}^{\frac{1}{\varphi}}\left\|_{L^{\varphi}} \leq \beta^{k}\right\|\left\{\sum_{j=1}^{\infty}\left(\frac{\kappa_{j} \chi_{Q_{j}}}{\left\|\chi_{Q_{j}}\right\|_{L^{\varphi}}}\right)^{\underline{\varphi}}\right\}^{\frac{1}{\varphi}} \|_{L^{\varphi}} .
$$

By virtue of the $\varphi$-triangle inequality, then we obtain

$$
\begin{aligned}
\left\|\left\{\sum_{j=1}^{\infty}\left(\frac{\kappa_{j}\left|f_{j}\right|^{\delta}\left|Q_{j}\right|^{\delta}}{\left\|f_{j}\right\|_{L^{1}\left(Q_{j}\right)}^{\delta}\left\|\chi_{Q_{j}}\right\|_{L^{\varphi}}}\right)^{\underline{\varphi}}\right\}^{\frac{1}{\varphi}}\right\|_{L^{\varphi}}^{\underline{\varphi}} & \leq\left\|\left\{\sum_{j=1}^{\infty} \sum_{k=0}^{\infty}\left(\frac{2^{\delta k(n+1)} \kappa_{j} \chi_{E_{j, k}}}{\left\|\chi_{Q_{j}}\right\|_{L^{\varphi}}}\right)^{\underline{\varphi}}\right\}^{\frac{1}{\varphi}}\right\|_{L^{\varphi}}^{\underline{\varphi}} \\
& \leq \sum_{k=0}^{\infty} 2^{\delta k(n+1) \underline{\varphi}}\left\|\left\{\sum_{j=1}^{\infty}\left(\frac{\kappa_{j} \chi_{E_{j, k}}}{\left\|\chi_{Q_{j}}\right\|_{L^{\varphi}}}\right)^{\underline{\varphi}}\right\}^{\underline{\underline{\varphi}}}\right\|_{L^{\varphi}}^{\underline{\varphi}} .
\end{aligned}
$$


Let $a=2^{\delta(n+1)} \beta$. Arithemetic shows that $a<1$; see (2.12). If we use (2.14), then we have

$$
\left\|\left\{\sum_{j=1}^{\infty}\left(\frac{\kappa_{j}\left|f_{j}\right|^{\delta}\left|Q_{j}\right|^{\delta}}{\left\|f_{j}\right\|_{L^{1}\left(Q_{j}\right)}^{\delta}\left\|\chi_{Q_{j}}\right\|_{L^{\varphi}}}\right)^{\underline{\varphi}}\right\}^{\frac{1}{\varphi}}\right\|_{L^{\varphi}}^{\underline{\varphi}} \leq \sum_{k=0}^{\infty} a^{k \underline{\varphi}}\left\|\left\{\sum_{j=1}^{\infty}\left(\frac{\kappa_{j} \chi_{Q_{j}}}{\left\|\chi_{Q_{j}}\right\|_{L^{\varphi}}}\right)^{\underline{\varphi}}\right\}^{\frac{1}{\varphi}}\right\|_{L^{\varphi}}^{\underline{\varphi}} .
$$

Thus, we conclude

$$
\begin{aligned}
\left\|\left\{\sum_{j=1}^{\infty}\left(\frac{\kappa_{j}\left|f_{j}\right|^{\delta}\left|Q_{j}\right|^{\delta}}{\left\|f_{j}\right\|_{L^{1}\left(Q_{j}\right)}^{\delta}\left\|\chi_{Q_{j}}\right\|_{L^{\varphi}}}\right)^{\underline{\varphi}}\right\}^{\frac{1}{\varphi}}\right\|_{L^{\varphi}} & \lesssim\left\|\left\{\sum_{j=1}^{\infty}\left(\frac{\kappa_{j} \chi_{Q_{j}}}{\left\|\chi_{Q_{j}}\right\|_{L^{\varphi}}}\right)^{\underline{\varphi}}\right\}^{\frac{1}{\varphi}}\right\|_{L^{\varphi}} \\
& =\mathcal{A}\left(\left\{\kappa_{j}\right\}_{j=1}^{\infty},\left\{Q_{j}\right\}_{j=1}^{\infty}\right) .
\end{aligned}
$$

Therefore, we obtain the desired result.

\subsection{Grand maximal operator and the moment condition}

Following [28], we recall the definition of the grand maximal operator $\mathcal{M}$.

Definition 2.17. Fix a large integer $N$.

1. Topologize $\mathcal{S}\left(\mathbb{R}^{n}\right)$, the set of all Schwartz functions, by the collection of seminorms $\left\{p_{\tilde{N}}\right\}_{\tilde{N} \in \mathbb{N}}$ given by

$$
p_{\tilde{N}}(\varphi) \equiv \sum_{|\alpha| \leq \tilde{N}} \sup _{x \in \mathbb{R}^{n}}(1+|x|)^{\tilde{N}}\left|\partial^{\alpha} \varphi(x)\right|
$$

for each $\tilde{N} \in \mathbb{N}$.

Define

$$
\mathcal{F}_{N} \equiv\left\{\varphi \in \mathcal{S}\left(\mathbb{R}^{n}\right): p_{N}(\varphi) \leq 1\right\}
$$

2. Let $f \in \mathcal{S}^{\prime}\left(\mathbb{R}^{n}\right)$ the set of all Schwartz distributions. Denote by $\mathcal{M} f$ the grand maximal operator given by

$$
\mathcal{M} f(x) \equiv \sup \left\{\left|t^{-n} \psi\left(t^{-1} \cdot\right) * f(x)\right|: t>0, \quad \psi \in \mathcal{F}_{N}\right\} \quad\left(x \in \mathbb{R}^{n}\right) .
$$

We recall how to use the moment condition here. In this paper, based on [26], we use the following notation.

Definition 2.18 (Moment condition).

1. Denote by $\mathcal{P}=\mathcal{P}\left(\mathbb{R}^{n}\right)$ the set of all polynomials. Then we can regard $\mathcal{P}\left(\mathbb{R}^{n}\right)$ canonically as the subset of $\mathcal{S}^{\prime}\left(\mathbb{R}^{n}\right)$. Denote by $\mathcal{P}_{d}\left(\mathbb{R}^{n}\right)$ the set of all polynomial functions with degree less than or equal to $d$, so that $\mathcal{P}\left(\mathbb{R}^{n}\right) \equiv$ $\bigcup_{d=0}^{\infty} \mathcal{P}_{d}\left(\mathbb{R}^{n}\right)$. It is understood that $\mathcal{P}_{-1}\left(\mathbb{R}^{n}\right)=\{0\}$. 
2. Let $L \in \mathbb{N}_{0}$. The set $\mathcal{P}_{L}\left(\mathbb{R}^{n}\right)^{\perp}$ denotes the set of all the measurable functions $f$ for which $(1+|\cdot|)^{L} f \in L^{1}\left(\mathbb{R}^{n}\right)$ and $\int_{\mathbb{R}^{n}} x^{\alpha} f(x) \mathrm{d} x=0$ for all $\alpha \in \mathbb{R}^{n}$ with $|\alpha| \leq L$. Such a function $f$ is said to satisfy the moment condition of order $L$. In this case, one also writes $f \perp \mathcal{P}_{L}\left(\mathbb{R}^{n}\right)$.

We state a lemma which will be used in Section 4. See [7, p. 598, B2] or [26, Theorem 1.55] for its proof.

Lemma 2.19. Let $N \in \mathbb{N}$ be a constant and let $\lambda \in(n+N, \infty)$. Also assume that $a \in C^{N}\left(\mathbb{R}^{n}\right)$ satisfies the differential inequality:

$$
\left|\partial^{\alpha} a(x)\right| \leq(1+|x|)^{-\lambda} \quad\left(x \in \mathbb{R}^{n}\right)
$$

for $|\alpha| \leq N$ and that $\eta \in \mathcal{P}_{N-1}\left(\mathbb{R}^{n}\right)^{\perp}$ satisfies the differential inequality:

$$
|\eta(x)| \leq(1+|x|)^{-\lambda} \quad\left(x \in \mathbb{R}^{n}\right) .
$$

For $j, \nu \in \mathbb{Z}$ satisfying $j \leq \nu$ we write $a^{j} \equiv 2^{j n} a\left(2^{j} \cdot\right), \eta^{\nu} \equiv 2^{\nu n} \eta\left(2^{\nu} \cdot\right)$. Then $\left|a^{j} * \eta^{\nu}(x)\right| \lesssim 2^{j n+(j-\nu) N}\left(1+\left|2^{j} x\right|\right)^{-\lambda}$ for all $x \in \mathbb{R}^{n}$.

We also invoke the following lemmas from [26, 28].

Lemma 2.20. [28] Let $f \in \mathcal{S}^{\prime}\left(\mathbb{R}^{n}\right), d \in\{0,1,2, \cdots\}$ and $j \in \mathbb{Z}$. Then there exist collections of cubes $\left\{Q_{j, k}\right\}_{k \in K_{j}}$ and functions $\left\{\eta_{j, k}\right\}_{k \in K_{j}} \subset C_{\text {comp }}^{\infty}\left(\mathbb{R}^{n}\right)$, which are all indexed by a set $K_{j}$ for every $j$, and a decomposition

$$
f=g_{j}+b_{j}, \quad b_{j}=\sum_{k \in K_{j}} b_{j, k}
$$

such that the following four conditions hold:

(i) Define $\mathcal{O}_{j} \equiv\left\{y \in \mathbb{R}^{n}: \mathcal{M} f(y)>2^{j}\right\}$ and consider its Whitney decomposition. Then the cubes $\left\{Q_{j, k}\right\}_{k \in K_{j}}$ satisfy

$$
\sum_{k \in K_{j}} \chi_{Q_{j, k}} \leq \chi_{\mathcal{O}_{j}} \leq \sum_{k \in K_{j}} \chi_{200 Q_{j, k}} \leq N \chi_{\mathcal{O}_{j}}
$$

for some $N \in \mathbb{N}$.

(ii) Consider the partition of unity with respect to $\left\{Q_{j, k}\right\}_{k \in K_{j}}$. Denote it by $\left\{\eta_{j, k}\right\}_{k \in K_{j}}$. Then each function $\eta_{j, k}$ is supported in $200 Q_{j, k}$ and

$$
\sum_{k \in K_{j}} \eta_{j, k}=\chi_{\mathcal{O}_{j}}=\chi_{\left(2^{j}, \infty\right]}(\mathcal{M} f), \quad 0 \leq \eta_{j, k} \leq 1 .
$$

(iii) The distribution $g_{j}$ satisfies

$$
\mathcal{M} g_{j}(x) \lesssim \mathcal{M} f(x) \chi_{\mathcal{O}_{j} c}(x)+2^{j} \sum_{k \in K_{j}} M \chi_{Q_{j, k}}(x)^{\frac{n+d+1}{n}}
$$

for all $x \in \mathbb{R}^{n}$. 
(iv) Each distribution $b_{j, k}$ is given by $b_{j, k}=\left(f-c_{j, k}\right) \eta_{j, k}$ with a certain polynomial $c_{j, k} \in \mathcal{P}_{d}\left(\mathbb{R}^{n}\right)$ satisfying

$$
\int_{\mathbb{R}^{n}} b_{j, k}(x) q(x) d \mathrm{x}=0 \text { for all } q \in \mathcal{P}_{d}\left(\mathbb{R}^{n}\right),
$$

and

$$
\mathcal{M} b_{j, k}(x) \lesssim \mathcal{M} f(x) \chi_{Q_{j, k}}(x)+2^{j} M \chi_{Q_{j, k}}(x)^{\frac{n+d+1}{n}}
$$

for all $x \in \mathbb{R}^{n}$.

In the above, $x_{j, k}$ and $\ell_{j, k}$ denote the center and the side-length of $Q_{j, k}$, respectively, and the implicit constants are dependent only on $n$.

Lemma 2.21. [26, Exercise 3.34] Fix $L \in \mathbb{N}_{0}$. Let $f \in H^{1}\left(\mathbb{R}^{n}\right)$. Then we can find $\left\{a_{j}\right\}_{j=1}^{\infty} \subset L^{\infty}\left(\mathbb{R}^{n}\right) \cap \mathcal{P}_{L}\left(\mathbb{R}^{n}\right)^{\perp}$ and a sequence $\left\{Q_{j}\right\}_{j=1}^{\infty}$ of cubes such that

1. $\operatorname{supp}\left(a_{j}\right) \subset Q_{j}$,

2. $f=\sum_{j=1}^{\infty} a_{j}$ in $\mathcal{S}^{\prime}\left(\mathbb{R}^{n}\right)$

3. For all $0<r<\infty,\left\{\sum_{j=1}^{\infty}\left(\left\|a_{j}\right\|_{\infty} \chi_{Q_{j}}\right)^{r}\right\}^{1 / r} \lesssim \mathcal{M} f$.

\section{Musielak-Orlicz Hardy space $H^{\varphi}\left(\mathbb{R}^{n}\right)$}

We are now oriented to Musielak-Orlicz Hardy spaces. We define Musielak-Orlicz Hardy spaces in Subsection 3.1. In Subsection 3.2, we give the definition of atomic Musielak-Orlicz Hardy spaces. To fix the language to describe the size of the coefficients, we use the quantity $\mathcal{A}\left(\left\{\kappa_{j}\right\}_{j=1}^{\infty},\left\{Q_{j}\right\}_{j=1}^{\infty}\right)$ dealt with in Subsection 2.2. We present the atomic characterization of Musielak-Orlicz Hardy spaces in Subsection 3.3, namely, atomic Musielak-Orlicz Hardy spaces and Musielak-Orlicz Hardy spaces defined in Subsection 3.1 are the same as a set.

\subsection{Musielak-Orlicz Hardy spaces by way of the grand maximal operators}

Following the spirit of [17], we define Musielak-Orlicz Hardy space $H^{\varphi}\left(\mathbb{R}^{n}\right)$ by means of the grand maximal function. The Musielak-Orlicz Hardy space $H^{\varphi}\left(\mathbb{R}^{n}\right)$ is the set of all $f \in \mathcal{S}^{\prime}\left(\mathbb{R}^{n}\right)$ for which the quantity

$$
\|f\|_{H^{\varphi}} \equiv\|\mathcal{M} f\|_{L^{\varphi}}
$$

is finite. As in [23, Theorem 3.4],

$$
\|f\|_{H^{\varphi}} \sim\left\|\sup _{t>0}\left|e^{t \Delta} f\right|\right\|_{L^{\varphi}} \quad\left(f \in \mathcal{S}^{\prime}\left(\mathbb{R}^{n}\right)\right) .
$$


Note that this generalizes the classical Hardy space $H^{p}\left(\mathbb{R}^{n}\right)$; simply take $\varphi(x, t)=$ $t^{p}$ for $(x, t) \in \mathbb{R}_{+}^{n+1}$.

We state a fundamental density result for Musielak-Orlicz Hardy spaces.

Lemma 3.1. Let $\varphi \in \mathbb{A}_{\infty}\left(\mathbb{R}^{n}\right)$ satisfy $0<i(\varphi) \leq I(\varphi)<\infty$. Then $H^{1}\left(\mathbb{R}^{n}\right) \cap$ $L^{2}\left(\mathbb{R}^{n}\right) \cap H^{\varphi}\left(\mathbb{R}^{n}\right)$ is dense in $H^{\varphi}\left(\mathbb{R}^{n}\right)$.

Proof. Use $g_{j}$ and $g_{-j}$ considered in Lemma 2.20 with $d \geq d_{\varphi}$. Since

$$
\begin{aligned}
\left\|\mathcal{M} g_{j}\right\|_{L^{\varphi}} & \lesssim\left\|\chi_{\left[0,2^{j}\right]}(\mathcal{M} f) \mathcal{M} f\right\|_{L^{\varphi}}+2^{j}\left\|\sum_{k \in K_{j}}\left(M \chi_{Q_{j, k}}\right)^{\frac{n+d+1}{n}}\right\|_{L^{\varphi}} \\
& \lesssim\left\|\chi_{\left[0,2^{j}\right]}(\mathcal{M} f) \mathcal{M} f\right\|_{L^{\varphi}}+2^{j}\left\|\sum_{k \in K_{j}} \chi_{Q_{j, k}}\right\|_{L^{\varphi}}
\end{aligned}
$$

thanks to (2.19) and Corollary 2.12. Recall that $\sum_{k \in K_{j}} \chi_{Q_{j, k}} \leq \chi_{\left(2^{j}, \infty\right]}(\mathcal{M} f)$ by Lemma 2.20 (i). Thus,

$$
\left\|\mathcal{M} g_{j}\right\|_{L^{\varphi}} \lesssim\left\|\min \left(\mathcal{M} f, 2^{j}\right)\right\|_{L^{\varphi}}(<\infty)
$$

Consequenlty, $f=\lim _{j \rightarrow-\infty}\left(f-g_{j}\right)$. Likewise, using (2.20), we can show that

$$
\left\|\mathcal{M}\left(f-g_{j}\right)\right\|_{L^{\varphi}} \lesssim\left\|\chi_{\left[2^{j}, \infty\right]}(\mathcal{M} f) \mathcal{M} f\right\|_{L^{\varphi}}<\infty .
$$

Thus, we conclude that $g_{j}-g_{-j} \in H^{\varphi}\left(\mathbb{R}^{n}\right)$ and $f=\lim _{j \rightarrow \infty}\left(g_{j}-g_{-j}\right)$ in $H^{\varphi}\left(\mathbb{R}^{n}\right)$. We claim that $g_{j}-g_{-j} \in H^{1}\left(\mathbb{R}^{n}\right) \cap L^{2}\left(\mathbb{R}^{n}\right)$ for each $j \in \mathbb{N}$. Indeed, if we fix $j \in \mathbb{N}$, we have

$$
\mathcal{M}\left(g_{j}\right)+\mathcal{M}\left(g_{-j}\right) \lesssim 2^{j}
$$

Meanwhile,

$$
\begin{aligned}
& \mathcal{M}\left(g_{j}-g_{-j}\right) \\
& =\mathcal{M}\left(b_{j}-b_{-j}\right) \\
& \lesssim \chi_{\left[2^{-j}, \infty\right]}(\mathcal{M} f) \mathcal{M} f+2^{j} \sum_{k \in K_{j}}\left(M \chi_{Q_{j, k}}\right)^{\frac{n+d+1}{n}}+2^{-j} \sum_{k \in K_{-j}}\left(M \chi_{Q_{-j, k}}\right)^{\frac{n+d+1}{n}} .
\end{aligned}
$$

Thus, since $\min (a+b+c, d) \leq \min (a+b+c, b+c+d)=\min (a, d)+b+c$ for all $a, b, c, d \geq 0$, from (3.1) and (3.2), we obtain

$$
\mathcal{M}\left(g_{j}-g_{-j}\right) \lesssim 2^{j}\left(\chi_{\left[2^{-j}, \infty\right]}(\mathcal{M} f)+\sum_{k \in K_{j}}\left(M \chi_{Q_{j, k}}\right)^{\frac{n+d+1}{n}}+\sum_{k \in K_{-j}}\left(M \chi_{Q_{-j, k}}\right)^{\frac{n+d+1}{n}}\right) .
$$

Since the right-hand side is integrable and square integrable, it follows that $g_{j}-$ $g_{-j} \in H^{1}\left(\mathbb{R}^{n}\right) \cap L^{2}\left(\mathbb{R}^{n}\right)$.

We remark that $H^{\varphi}\left(\mathbb{R}^{n}\right)$ and $L^{\varphi}\left(\mathbb{R}^{n}\right)$ if $q(\varphi)<i(\varphi) \leq I(\varphi)<\infty$, which transforms many results in this paper into the ones for Musielak-Orlicz spaces. 
Theorem 3.2. Suppose that $\varphi \in A_{\infty}\left(\mathbb{R}^{n}\right)$ satisfies $q(\varphi)<i(\varphi) \leq I(\varphi)<\infty$. Then $L^{\varphi}\left(\mathbb{R}^{n}\right) \approx H^{\varphi}\left(\mathbb{R}^{n}\right)$ with the equivalence of norms.

Proof. Thanks to Lemma 2.11, we have $L^{\varphi}\left(\mathbb{R}^{n}\right)$ is a subset of $H^{\varphi}\left(\mathbb{R}^{n}\right)$. Let us prove the opposite inclusion. Let $f \in H^{\varphi}\left(\mathbb{R}^{n}\right)$. Then there exists $\left\{f_{j}\right\}_{j=1}^{\infty} \in$ $H^{\varphi}\left(\mathbb{R}^{n}\right) \cap L^{2}\left(\mathbb{R}^{n}\right) \cap H^{1}\left(\mathbb{R}^{n}\right)$ such that $f_{j} \rightarrow f$ in $H^{\varphi}\left(\mathbb{R}^{n}\right)$ thanks to Lemma 3.1. Since $f_{j}-f_{k} \in L^{2}\left(\mathbb{R}^{n}\right) \subset L_{\text {loc }}^{1}\left(\mathbb{R}^{n}\right)$ we have $\left|f_{j}-f_{k}\right| \leq \sup _{t>0}\left|e^{t \Delta}\left[f_{j}-f_{k}\right]\right|$. Consequently, $\left\|f_{j}-f_{k}\right\|_{L^{\varphi}} \leq\left\|f_{j}-f_{k}\right\|_{H^{\varphi}}$. Since $\left\{f_{j}\right\}_{j=1}^{\infty}$ converges to $f$ in $H^{\varphi}\left(\mathbb{R}^{n}\right),\left\{f_{j}\right\}_{j=1}^{\infty}$ is a Cauchy sequence in $L^{\varphi}\left(\mathbb{R}^{n}\right)$. Meanwhile, since $L^{\varphi}\left(\mathbb{R}^{n}\right)$ is embedded into $L_{\text {loc }}^{1}\left(\mathbb{R}^{n}\right)$ owing to Lemma 2.11 again, it follows that $f$ is the limit of $\left\{f_{j}\right\}_{j=1}^{\infty}$ in the topology of $L^{\varphi}\left(\mathbb{R}^{n}\right)$. Thus, $f \in L^{\varphi}\left(\mathbb{R}^{n}\right)$.

\subsection{Atomic Musielak-Orlicz Hardy spaces}

We are now oriented to another definition of Musielak-Orlicz Hardy spaces. We define Musielak-Orlicz Hardy spaces by using atoms. Here we present the definition of atoms.

Definition 3.3. Let $1<u \leq \infty, \varphi \in \mathbb{A}_{\infty}\left(\mathbb{R}^{n}\right)$ with $0<i(\varphi) \leq I(\varphi)<\infty$ and let $d \in\left[d_{\varphi}, \infty\right) \cap \mathbb{Z}$. An $L^{u}$-function $a$ is said to be a $(\varphi, u)$-atom supported on $Q$ if it is supported on a cube $Q$ with the following properties.

1. $\operatorname{supp}(a) \subset Q$.

2. $\|a\|_{L^{u}} \leq \frac{|Q|^{\frac{1}{u}}}{\left\|\chi_{Q}\right\|_{L^{\varphi}}}$.

3. $a \in \mathcal{P}_{d}\left(\mathbb{R}^{n}\right)^{\perp}$.

The set $\mathcal{A}(\varphi, u)$ collects all $\left\{\left(a_{j}, Q_{j}\right)\right\}_{j=1}^{\infty}$ such that each $a_{j}$ is a $(\varphi, u)$-atom supported on $Q_{j}$.

A direct consequence from the definition is

$$
\mathcal{M} a \lesssim \chi_{2 \sqrt{n} Q} M a+\frac{1}{\left\|\chi_{Q}\right\|_{L^{\varphi}}}\left(M \chi_{Q}\right)^{\frac{n+d+1}{n}} .
$$

See the proof of [23, Proposition 5.3].

Definition $3.4\left(H_{\text {atom }}^{\varphi, u}\left(\mathbb{R}^{n}\right)\right)$. Let $\varphi \in \mathbb{A}_{\infty}\left(\mathbb{R}^{n}\right)$ satisfy $0<i(\varphi) \leq I(\varphi)<\infty$ and $1<u \leq \infty$. The atomic Musielak-Orlicz Hardy space $H_{\text {atom }}^{\varphi, u}\left(\mathbb{R}^{n}\right)$ is the set of all functions $f \in \mathcal{S}^{\prime}\left(\mathbb{R}^{n}\right)$ such that it can be written as

$$
f=\sum_{j=1}^{\infty} \kappa_{j} a_{j} \text { in } \mathcal{S}^{\prime}\left(\mathbb{R}^{n}\right),
$$

where $\left\{\kappa_{j}\right\}_{j=1}^{\infty}$ is a sequence of nonnegative numbers, $\left\{\left(a_{j}, Q_{j}\right)\right\}_{j=1}^{\infty} \subset \mathcal{A}(\varphi, u)$ and $\mathcal{A}\left(\left\{\kappa_{j}\right\}_{j=1}^{\infty},\left\{Q_{j}\right\}_{j=1}^{\infty}\right)$ is finite. One defines

$$
\|f\|_{H_{\text {atom }}^{\varphi, u}} \equiv \inf \mathcal{A}\left(\left\{\kappa_{j}\right\}_{j=1}^{\infty},\left\{Q_{j}\right\}_{j=1}^{\infty}\right),
$$

where the infimum is taken over all admissible expressions as in (3.4). 
It should be noted that $H_{\text {atom }}^{\varphi, u}\left(\mathbb{R}^{n}\right)$ implicitly depends on $d \in\left[d_{\varphi}, \infty\right) \cap \mathbb{Z}$. However, as we will see in Theorem 3.6, the definition of $H_{\text {atom }}^{\varphi, u}\left(\mathbb{R}^{n}\right)$ does not depend on $d \in\left[d_{\varphi}, \infty\right) \cap \mathbb{Z}$ as a set; the different choices of $d$ will yield equivalent norms. It is also noteworthy that

$$
H_{\text {atom }}^{\varphi, \infty}\left(\mathbb{R}^{n}\right) \hookrightarrow H_{\text {atom }}^{\varphi, u}\left(\mathbb{R}^{n}\right)
$$

thanks to Hölder's inequaltiy.

It is useful to torelate the case where each atom has non-compact support. To justify this idea, we will use the notion of molecules.

Definition 3.5. Let $\varphi \in \mathbb{A}_{\infty}\left(\mathbb{R}^{n}\right)$ satisfy $0<i(\varphi) \leq I(\varphi)<\infty, 1<u \leq \infty$ and let $d \in\left[d_{\varphi}, \infty\right) \cap \mathbb{Z}$. An $L^{u}$-function $\mathfrak{M}$ is said to be a $(\varphi, u)$-molecule supported on $Q$ if there is a cube $Q$ with the following properties.

1. $\left\|\chi_{2 \sqrt{n} Q} \mathfrak{M}\right\|_{L^{u}} \leq \frac{|Q|^{\frac{1}{u}}}{\left\|\chi_{Q}\right\|_{L^{\varphi}}}$

2. $\chi_{\mathbb{R}^{n} \backslash 2 \sqrt{n} Q}|\mathfrak{M}| \leq\left(M \chi_{Q}\right)^{\frac{2 n+2 d+3}{n}}$.

3. $\mathfrak{M} \in \mathcal{P}_{d}\left(\mathbb{R}^{n}\right)^{\perp}$.

The set $\mathcal{M}(\varphi, u)$ collects all $\left\{\left(a_{j}, Q_{j}\right)\right\}_{j=1}^{\infty}$ such that each $a_{j}$ is a $(\varphi, u)$-molecule supported on $Q_{j}$.

As in $[23,(5.2)]$, we have

$$
\sup _{t>0}\left|e^{t \Delta} \mathfrak{M}\right| \lesssim \chi_{2 \sqrt{n} Q} M \mathfrak{M}+\frac{1}{\left\|\chi_{Q}\right\|_{L^{\varphi}}}\left(M \chi_{Q}\right)^{\frac{n+d+1}{n}} .
$$

We can define $H_{\text {molecule }}^{\varphi, u}\left(\mathbb{R}^{n}\right)$ analogously to $H_{\text {atom }}^{\varphi, u}\left(\mathbb{R}^{n}\right)$ by replacing atoms by molecule in Definition 3.4. It is trivial that $H_{\text {atom }}^{\varphi, u}\left(\mathbb{R}^{n}\right) \hookrightarrow H_{\text {molecule }}^{\varphi, u}\left(\mathbb{R}^{n}\right)$. However, it turns out to be the same space; see Remark 3.10.

\subsection{Atomic characterization of Musielak-Orlicz Hardy spaces}

Theorem 3.6 below is one of the key results in this paper. In analogy to the results in [23], we can prove the following result.

Theorem 3.6. Let $\varphi \in \mathbb{A}_{\infty}\left(\mathbb{R}^{n}\right)$ satisfy $0<i(\varphi) \leq I(\varphi)<\infty, 1 \ll u \leq \infty$ and let $d \in\left[d_{\varphi}, \infty\right) \cap \mathbb{Z}$. Then $H_{\text {atom }}^{\varphi, u}\left(\mathbb{R}^{n}\right) \approx H^{\varphi}\left(\mathbb{R}^{n}\right)$ with equivalence of norms. In particular, the definition of $H_{\text {atom }}^{\varphi, u}\left(\mathbb{R}^{n}\right)$ does not depend on $d \in\left[d_{\varphi}, \infty\right) \cap \mathbb{Z}$ as a set; the different choices of $d$ will yield equivalent norms.

Proof. Fix an integer $d \geq d_{\varphi}$. Let $f \in H_{\text {atom }}^{\varphi, u}\left(\mathbb{R}^{n}\right)$. Then we have a decomposition:

$$
f=\sum_{j=1}^{\infty} \kappa_{j} a_{j},
$$


where $\left\{\kappa_{j}\right\}_{j=1}^{\infty}$ is a sequence of nonnegative numbers, $\left\{\left(a_{j}, Q_{j}\right)\right\}_{j=1}^{\infty} \subset \mathcal{A}(\varphi, u)$ and $\mathcal{A}\left(\left\{\kappa_{j}\right\}_{j=1}^{\infty},\left\{Q_{j}\right\}_{j=1}^{\infty}\right)<\infty$. By $(3.3)$,

$$
\mathcal{M} f \lesssim \sum_{j=1}^{\infty} \kappa_{j}\left(\chi_{2 \sqrt{n} Q_{j}} M a_{j}+\frac{1}{\left\|\chi_{Q_{j}}\right\|_{L^{\varphi}}}\left(M \chi_{Q_{j}}\right)^{\frac{n+d+1}{n}}\right) .
$$

Let $u<\infty$. Since $M$ is bounded on $L^{u}\left(\mathbb{R}^{n}\right)$, we have

$$
\left\|M a_{j}\right\|_{L^{u}\left(2 \sqrt{n} Q_{j}\right)} \lesssim\left\|a_{j}\right\|_{L^{u}\left(Q_{j}\right)} \leq \frac{\left|Q_{j}\right|^{1 / u}}{\left\|\chi_{Q_{j}}\right\|_{L^{\varphi}}}
$$

and hence

$$
\chi_{2 \sqrt{n} Q_{j}} M a_{j} \lesssim \frac{\left|Q_{j}\right|^{1 / u} \chi_{2 \sqrt{n} Q_{j}} M a_{j}}{\left\|M a_{j}\right\|_{L^{u}\left(2 \sqrt{n} Q_{j}\right)}\left\|\chi_{Q_{j}}\right\|_{L^{\varphi}}} .
$$

We take the $L^{\varphi}$-norm to have

$$
\|\mathcal{M} f\|_{L^{\varphi}} \lesssim\left\|\sum_{j=1}^{\infty} \kappa_{j} \chi_{2 \sqrt{n} Q_{j}} M a_{j}\right\|_{L^{\varphi}}+\left\|\sum_{j=1}^{\infty} \frac{\kappa_{j}}{\left\|\chi_{Q_{j}}\right\|_{L^{\varphi}}}\left(M \chi_{Q_{j}}\right)^{\frac{n+d+1}{n}}\right\|_{L^{\varphi}}
$$

If we use Lemma 2.11 to have

$$
\|\mathcal{M} f\|_{L^{\varphi}} \lesssim\left\|\sum_{j=1}^{\infty} \kappa_{j} \frac{\left|Q_{j}\right|^{1 / u} \chi_{2 \sqrt{n} Q_{j}} M a_{j}}{\left\|M a_{j}\right\|_{L^{u}\left(2 \sqrt{n} Q_{j}\right)}\left\|\chi_{Q_{j}}\right\|_{L^{\varphi}}}\right\|_{L^{\varphi}}+\left\|\sum_{j=1}^{\infty} \frac{\kappa_{j}}{\left\|\chi_{Q_{j}}\right\|_{L^{\varphi}}} \chi_{Q_{j}}\right\|_{L^{\varphi}}
$$

By the embedding $\ell^{1}(\mathbb{N}) \hookrightarrow \ell \underline{\varphi}(\mathbb{N})$, we obtain

$$
\begin{aligned}
& \|\mathcal{M} f\|_{L^{\varphi}} \\
& \lesssim\left\|\left\{\sum_{j=1}^{\infty}\left(\kappa_{j} \frac{\left|Q_{j}\right|^{1 / u} \chi_{2 \sqrt{n} Q_{j}} M a_{j}}{\left\|M a_{j}\right\|_{L^{u}\left(2 \sqrt{n} Q_{j}\right)}\left\|\chi_{Q_{j}}\right\|_{L^{\varphi}}}\right)^{\underline{\varphi}}\right\}^{\frac{1}{\varphi}}\right\|_{L^{\varphi}}+\left\|\left\{\sum_{j=1}^{\infty}\left(\frac{\kappa_{j}}{\left\|\chi_{Q_{j}}\right\|_{L^{\varphi}}} \chi_{Q_{j}}\right)^{\underline{\varphi}}\right\}^{\frac{1}{\varphi}}\right\|_{L^{\varphi}} .
\end{aligned}
$$

If we use Lemmas 2.11 and 2.16 as well as (2.7), then we obtain

$$
\|\mathcal{M} f\|_{L^{\varphi}} \lesssim\left\|\left\{\sum_{j=1}^{\infty}\left(\frac{\kappa_{j}}{\left\|\chi_{Q_{j}}\right\|_{L^{\varphi}}} \chi_{2 \sqrt{n} Q_{j}}\right)^{\underline{\varphi}}\right\}^{\frac{1}{\varphi}}\right\|_{L^{\varphi}} \lesssim \mathcal{A}\left(\left\{\kappa_{j}\right\}_{j=1}^{\infty},\left\{Q_{j}\right\}_{j=1}^{\infty}\right) .
$$

A similar conclusion to (3.9) also holds for $u=\infty$ since

$$
\chi_{2 \sqrt{n} Q_{j}} M a_{j} \leq \chi_{2 \sqrt{n} Q_{j}}\left\|a_{j}\right\|_{L^{\infty}} \leq \chi_{2 \sqrt{n} Q_{j}} \frac{1}{\left\|\chi_{Q_{j}}\right\|_{L^{\varphi}}} .
$$

Thus, $f \in H^{\varphi}\left(\mathbb{R}^{n}\right)$.

Conversely we will show that $H^{\varphi}\left(\mathbb{R}^{n}\right) \hookrightarrow H_{\text {atom }}^{\varphi, u}\left(\mathbb{R}^{n}\right)$. Thanks to (3.5), we may assume $u=\infty$. We will show that $f \in H_{\text {atom }}^{\varphi, u}\left(\mathbb{R}^{n}\right)$ and that

$$
\|f\|_{H_{\mathrm{atom}}^{\varphi, u}} \lesssim\|\mathcal{M} f\|_{L^{\varphi}}
$$

We may assume $f \in H^{\varphi}\left(\mathbb{R}^{n}\right) \cap H^{1}\left(\mathbb{R}^{n}\right) \cap L^{2}\left(\mathbb{R}^{n}\right)$ in view of Lemma 3.1.

If we use Lemma 2.21, we can find $\left\{A_{j}\right\}_{j=1}^{\infty} \subset L^{\infty}\left(\mathbb{R}^{n}\right) \cap \mathcal{P}_{d}\left(\mathbb{R}^{n}\right)^{\perp}$ and a sequence $\left\{Q_{j}\right\}_{j=1}^{\infty}$ of cubes such that 
1. $A_{j} \neq 0$,

2. $\operatorname{supp}\left(A_{j}\right) \subset Q_{j}$,

3. $f=\sum_{j=1}^{\infty} A_{j}$ in $\mathcal{S}^{\prime}\left(\mathbb{R}^{n}\right)$,

4. For all $0<r<\infty,\left\{\sum_{j=1}^{\infty}\left(\left\|A_{j}\right\|_{\infty} \chi_{Q_{j}}\right)^{r}\right\}^{1 / r} \lesssim \mathcal{M} f$.

Thus, if we let

$$
r=\underline{\varphi}, \quad \lambda_{j}=\left\|A_{j}\right\|_{L^{\infty}}\left\|\chi_{Q_{j}}\right\|_{L^{\varphi}}, \quad a_{j}=\frac{1}{\lambda_{j}} A_{j},
$$

then we obtain

$$
f=\sum_{j=1}^{\infty} \lambda_{j} a_{j}
$$

and

$\left\|\left\{\sum_{j=1}^{\infty}\left(\frac{\lambda_{j} \chi_{Q_{j}}}{\left\|\chi_{Q_{j}}\right\|_{L^{\varphi}}}\right)^{\underline{\varphi}}\right\}^{1 / \underline{\varphi}}\right\|_{L^{\varphi}}=\left\|\left\{\sum_{j=1}^{\infty}\left(\left\|A_{j}\right\|_{\infty} \chi_{Q_{j}}\right)^{\underline{\varphi}}\right\}^{1 / \underline{\varphi}}\right\|_{L^{\varphi}} \lesssim\|\mathcal{M} f\|_{L^{\varphi}}=\|f\|_{H^{\varphi}}$.

Thus, $f \in H_{\text {atom }}^{\varphi, \infty}\left(\mathbb{R}^{n}\right)$.

We combine Theorems 3.2 and 3.6 to have the atomic characterization of Musielak-Orlicz spaces.

Theorem 3.7. Let $\varphi \in \mathbb{A}_{\infty}\left(\mathbb{R}^{n}\right)$ satisfy $q(\varphi)<i(\varphi) \leq I(\varphi)<\infty, 1 \ll u \leq \infty$ and let $d \in\left[d_{\varphi}, \infty\right) \cap \mathbb{Z}$. Then $H_{\text {atom }}^{\varphi, u}\left(\mathbb{R}^{n}\right) \approx L^{\varphi}\left(\mathbb{R}^{n}\right)$ with equivalence of norms.

The inclusion $L^{\varphi}\left(\mathbb{R}^{n}\right) \hookrightarrow H_{\text {atom }}^{\varphi, u}\left(\mathbb{R}^{n}\right)$ as well as Lemma 2.16 is useful. Here we explicitly describe one of the important assertions of Theorem 3.7.

Corollary 3.8. Let $\varphi \in \mathbb{A}_{\infty}\left(\mathbb{R}^{n}\right)$ satisfy $q(\varphi)<i(\varphi) \leq I(\varphi)<\infty, 1 \ll u \leq \infty$ and let $d \in\left[d_{\varphi}, \infty\right) \cap \mathbb{Z}$. Then any $f \in L^{\varphi}\left(\mathbb{R}^{n}\right)$ can be written in the form (3.4), where $\left\{\kappa_{j}\right\}_{j=1}^{\infty}$ is a sequence of nonnegative numbers, $\left\{\left(a_{j}, Q_{j}\right)\right\}_{j=1}^{\infty} \subset \mathcal{A}(\varphi, u)$ and $\mathcal{A}\left(\left\{\kappa_{j}\right\}_{j=1}^{\infty},\left\{Q_{j}\right\}_{j=1}^{\infty}\right) \lesssim\|f\|_{L^{\varphi}}$.

Before we conclude this section, we make helpful remarks.

Remark 3.9. If $f \in H^{\varphi}\left(\mathbb{R}^{n}\right) \cap L^{2}\left(\mathbb{R}^{n}\right)$, then convergence of (3.10) takes place in $H^{\varphi}\left(\mathbb{R}^{n}\right) \cap L^{2}\left(\mathbb{R}^{n}\right)$. In fact, let

$$
f_{N}=\sum_{j=1}^{N} \lambda_{j} a_{j} \quad(N \in \mathbb{N})
$$


Then due to (3.9),

$$
\left\|f-f_{N}\right\|_{H^{\varphi}} \lesssim \mathcal{A}_{\varphi}\left(\left\{\kappa_{j}\right\}_{j=N+1}^{\infty},\left\{Q_{j}\right\}_{j=N+1}^{\infty}\right) \rightarrow 0 \quad(N \rightarrow \infty)
$$

and

$$
\left\|f-f_{N}\right\|_{L^{2}} \sim\left\|f-f_{N}\right\|_{H^{2}} \lesssim \mathcal{A}_{2}\left(\left\{\kappa_{j}\right\}_{j=N+1}^{\infty},\left\{Q_{j}\right\}_{j=N+1}^{\infty}\right) \rightarrow 0 \quad(N \rightarrow \infty),
$$

as required.

Remark 3.10. Let $\varphi \in \mathbb{A}_{\infty}\left(\mathbb{R}^{n}\right)$ satisfy $0<i(\varphi) \leq I(\varphi)<\infty, 1<u \leq \infty$. Remark that $H^{\varphi}\left(\mathbb{R}^{n}\right) \approx H_{\text {atom }}^{\varphi, u}\left(\mathbb{R}^{n}\right) \approx H_{\text {molecule }}^{\varphi, u}\left(\mathbb{R}^{n}\right)$ with equivalence of norms. In fact, in view of Theorem 3.6, what remains unclear is $H_{\text {molecule }}^{\varphi, u}\left(\mathbb{R}^{n}\right) \hookrightarrow H^{\varphi}\left(\mathbb{R}^{n}\right)$. However, this is a consequence of (3.6); see the proof of $H_{\text {atom }}^{\varphi, u}\left(\mathbb{R}^{n}\right) \hookrightarrow H^{\varphi}\left(\mathbb{R}^{n}\right)$, where (3.3) is employed in (3.7).

Finally, to conclude this section, we will show that our atomic characterization readily yields the decomposition obtained in [17, Section 5] assuming that $\varphi$ is a growth function. Following [17], we recall the known results on the atomic characterization of Musielak-Orlicz Hardy spaces.

Definition 3.11. Let $\varphi \in \mathbb{A}_{\infty}\left(\mathbb{R}^{n}\right)$ be a growth function, namely $0<i(\varphi) \leq$ $I(\varphi) \leq 1$.

1. For a measurable set $E$, we write $\varphi(E, t)=\int_{E} \varphi(x, t) d \mathrm{x}$.

2. For a cube $Q$ and $1 \leq u<\infty$, we denote by $L_{\varphi}^{u}(Q)$ the set of all measurable functions $f$ on $\mathbb{R}^{n}$ supported in $Q$ such that

$$
\|f\|_{L_{\varphi}^{u}}=\sup _{t>0}\left(\frac{1}{\varphi(Q, t)} \int_{\mathbb{R}^{n}}|f(x)|^{u} \varphi(x, t) d \mathrm{x}\right)^{\frac{1}{u}}<\infty .
$$

3. Let $1 \leq u<\infty$. Let $\left\{a_{j}\right\}_{j=1}^{\infty}$ be a sequence of $(\varphi, u)$-atoms. Denote by $\left\{Q_{j}\right\}_{j=1}^{\infty}$ the corresponding sequence of cubes. Let also $\left\{\kappa_{j}\right\}_{j=1}^{\infty}$ be a nonnegative sequence of real numbers. Then define

$$
\Lambda_{u}\left(\left\{a_{j}\right\}_{j=1}^{\infty},\left\{\kappa_{j}\right\}_{j=1}^{\infty}\right)=\inf \left\{\lambda>0: \sum_{j=1}^{\infty} \int_{Q_{j}} \varphi\left(x, \frac{\left\|\kappa_{j} a_{j}\right\|_{L_{\varphi}^{u}}}{\lambda}\right) \leq 1\right\} .
$$

4. Let $\left\{a_{j}\right\}_{j=1}^{\infty}$ be a sequence of $(\varphi, \infty)$-atoms. Denote by $\left\{Q_{j}\right\}_{j=1}^{\infty}$ the corresponding sequence of cubes. Then define

$$
\Lambda_{\infty}\left(\left\{a_{j}\right\}_{j=1}^{\infty},\left\{\kappa_{j}\right\}_{j=1}^{\infty}\right)=\inf \left\{\lambda>0: \sum_{j=1}^{\infty} \int_{Q_{j}} \varphi\left(x, \frac{\kappa_{j}}{\lambda\left\|\chi_{Q_{j}}\right\|_{L^{\varphi}}}\right) \leq 1\right\}
$$

5. Let $1 \leq u<\infty$. For $f \in H^{\varphi}\left(\mathbb{R}^{n}\right)$, define

$$
\|f\|_{\tilde{H}_{\mathrm{atom}}^{\varphi, u}}=\inf _{(3.4)} \Lambda_{u}\left(\left\{a_{j}\right\}_{j=1}^{\infty},\left\{\lambda_{j}\right\}_{j=1}^{\infty}\right),
$$

where $\left\{a_{j}\right\}_{j=1}^{\infty}$ and $\left\{\lambda_{j}\right\}_{j=1}^{\infty}$ move over all decomposition of $f$ as in (3.4). 
By Hölder's inequality, $\|f\|_{\tilde{H}_{\text {atom }}^{\varphi, u_{1}}} \leq\|f\|_{\tilde{H}_{\text {atom }}^{\varphi, u_{2}}}$ if $1<u_{1}<u_{2} \leq \infty$.

Theorem 3.12. Let $\varphi \in \mathbb{A}_{\infty}\left(\mathbb{R}^{n}\right)$ be a growth function satisfying $0<i(\varphi) \leq$ $I(\varphi) \leq 1$, and let $1 \ll u \leq \infty$. Then the norms $\|f\|_{\tilde{H}_{\text {atom }}^{\varphi, u}}$ and $\|f\|_{H^{\varphi}}$ are equivalent for all $f \in H^{\varphi}\left(\mathbb{R}^{n}\right)$.

Proof. Due to Lemma 2.7, we have $\Lambda_{u}(\cdot, \cdot) \leq \Lambda_{\infty}(\cdot, \cdot) \leq \mathcal{A}_{\infty}(\cdot, \cdot)$. Hence by Theorem 3.6, we have $\|f\|_{\tilde{H}_{\text {atom }}^{\varphi, u}} \lesssim\|f\|_{H_{\text {atom }}^{\varphi, u}} \lesssim\|f\|_{H^{\varphi}}$. For the opposite inequality, we use [17, Theorem 5.2].

\section{Generalized singular integral operators}

As an application of Theorem 3.6, we will prove the boundedness of generalized singular integral operators of order $s$. An $L^{2}$-bounded linear operator $T$ is a (generalized) singular integral operator (with the kernel $K \in L_{\text {loc }}^{1}\left(\mathbb{R}^{n} \times \mathbb{R}^{n} \backslash\{(x, x)\right.$ : $\left.x \in \mathbb{R}^{n}\right\}$ ) of order $s$ ), if it satisfies the following conditions:

(1) For all $f \in L^{2}\left(\mathbb{R}^{n}\right)$ with compact support, we have

$$
T f(x)=\int_{\mathbb{R}^{n}} K(x, y) f(y) \mathrm{d} y \text { for almost all } x \notin \operatorname{supp}(f) .
$$

(2) For all $x, y \in \mathbb{R}^{n}$ such that $x \neq y$, size condition:

$$
\left|\partial_{x}^{\alpha} \partial_{y}^{\beta} K(x, y)\right| \lesssim|x-y|^{-n-|\alpha|-|\beta|}
$$

holds for all $|\alpha|,|\beta| \leq s$.

As we did in [23, p. 3700], we can prove the following:

Lemma 4.1. Let $\varphi \in \mathbb{A}_{\infty}\left(\mathbb{R}^{n}\right)$ satisfy $0<i(\varphi) \leq I(\varphi)<\infty, 1<u \leq \infty$ and let $s \in\left[d_{\varphi}, \infty\right) \cap \mathbb{Z}$. Let also $T$ be a singular integral operator with the kernel $K \in L_{\text {loc }}^{1}\left(\mathbb{R}^{n} \times \mathbb{R}^{n} \backslash\left\{(x, x): x \in \mathbb{R}^{n}\right\}\right)$ of order $s$. Then for any $(\varphi, u)$-atom a with the cube $Q$,

$$
|T a| \lesssim|T a| \chi_{2 \sqrt{n} Q}+\frac{1}{\left\|\chi_{Q}\right\|_{L^{\varphi}}}\left(M \chi_{Q}\right)^{\frac{n+s+1}{n}}
$$

Similar to [23, Proposition 5.3], a direct consequence of this observation and the density result, Lemma 3.1, is the following conclusion.

Theorem 4.2. Let $\varphi \in \mathbb{A}_{\infty}\left(\mathbb{R}^{n}\right)$ satisfy $0<i(\varphi) \leq I(\varphi)<\infty$. Then any singular integral operator $T$ with the kernel $K \in L_{\text {loc }}^{1}\left(\mathbb{R}^{n} \times \mathbb{R}^{n} \backslash\left\{(x, x): x \in \mathbb{R}^{n}\right\}\right)$ of order $d_{\varphi}$, which is initially defined on $L^{2}\left(\mathbb{R}^{n}\right)$, extends to a bounded linear operator from $H^{\varphi}\left(\mathbb{R}^{n}\right)$ to $L^{\varphi}\left(\mathbb{R}^{n}\right)$.

Proof. We have only to prove that $T$ is bounded extends to a bounded linear operator from $H_{\text {atom }}^{\varphi, \infty}\left(\mathbb{R}^{n}\right)$ to $L^{\varphi}\left(\mathbb{R}^{n}\right)$ : we have only to show that $\|T f\|_{L^{\varphi}} \lesssim\|f\|_{H_{\text {atom }}^{\varphi, \infty}}$. for all $f \in H_{\text {atom }}^{\varphi, \infty}\left(\mathbb{R}^{n}\right)$. Thanks to Lemma 3.1 and Theorem 3.6, we may assume 
$f \in H_{\text {atom }}^{\varphi, \infty}\left(\mathbb{R}^{n}\right) \cap L^{2}\left(\mathbb{R}^{n}\right)$. Then, owing to Remark 3.9, $f$ can be written as $f=$ $\sum_{j=1}^{\infty} \kappa_{j} a_{j}$ in $L^{2}\left(\mathbb{R}^{n}\right)$, where $\left\{\kappa_{j}\right\}_{j=1}^{\infty} \subset[0, \infty),\left\{\left(a_{j}, Q_{j}\right)\right\}_{j=1}^{\infty} \subset \mathcal{A}(\varphi, \infty)$ and

$$
\mathcal{A}\left(\left\{\kappa_{j}\right\}_{j=1}^{\infty},\left\{Q_{j}\right\}_{j=1}^{\infty}\right) \leq 2\|f\|_{H_{\mathrm{atom}}^{\varphi, \infty}}
$$

Then, $T f=\sum_{j=1}^{\infty} \kappa_{j} T a_{j}$ in $L^{2}\left(\mathbb{R}^{n}\right)$. Thanks to Lemma 4.1,

$$
|T f| \leq \sum_{j=1}^{\infty} \kappa_{j}\left(\left|T a_{j}\right| \chi_{2 \sqrt{n} Q_{j}}+\frac{1}{\left\|\chi_{Q}\right\|_{L^{\varphi}}}\left(M \chi_{Q}\right)^{\frac{n+d_{\varphi}+1}{n}}\right) .
$$

If we use Lemma 2.16 and (2.7) and argue similarly to Theorem 3.6, we obtain

$$
\begin{aligned}
\|T f\|_{L^{\varphi}} & \lesssim\left\|\left(\sum_{j=1}^{\infty}\left(\frac{\kappa_{j}\left|Q_{j}\right|^{1 / u}\left|T a_{j}\right| \chi_{2 \sqrt{n} Q_{j}}}{\left\|T a_{j}\right\|_{L^{u}\left(2 \sqrt{n} Q_{j}\right)}\left\|\chi_{Q_{j}}\right\|_{L^{u}}}\right)^{\underline{\varphi}}\right)^{1 / \underline{\varphi}}\right\|_{L^{\varphi}}+\left\|\sum_{j=1}^{\infty} \frac{\kappa_{j}}{\left\|\chi_{Q_{j}}\right\|_{L^{\varphi}}} \chi_{Q_{j}}\right\|_{L^{\varphi}} \\
& \leq \mathcal{A}\left(\left\{\kappa_{j}\right\}_{j=1}^{\infty},\left\{Q_{j}\right\}_{j=1}^{\infty}\right) \\
& \leq 2\|f\|_{H_{\text {atom }}^{\varphi, \infty}}
\end{aligned}
$$

as required.

Remark 4.3. In addition to the assumption in Theorem 4.2, assume that any $(\varphi, \infty)$-atom is transformed into $(\varphi, u)$-molecules modulo a multiplicative constant. Namely, we suppose that there exists a constant $D_{u}>0$ depending only on $D$ such that $D^{-1} T a$ is a $(\varphi, u)$-molecule for any $(\varphi, \infty)$-atom $a$. Then $T$, which is initially defined on $L^{2}\left(\mathbb{R}^{n}\right)$, extends to a bounded linear operator on $H^{\varphi}\left(\mathbb{R}^{n}\right)$. This is a consequence of Theorem 3.6 and Remark 3.10.

As an application of Remark 4.3, in analogy to [23, Theorem 5.7], we obtain the following characterization:

Theorem 4.4. Let $\varphi \in \mathbb{A}_{\infty}\left(\mathbb{R}^{n}\right)$ satisfy $0<i(\varphi) \leq I(\varphi)<\infty$. Let $\tau \in \mathcal{S}\left(\mathbb{R}^{n}\right)$ satisfy $\chi_{B(4) \backslash B(2)} \leq \tau \leq \chi_{B(8) \backslash B(1)}$. Then for $f \in \mathcal{S}^{\prime}\left(\mathbb{R}^{n}\right)$, we have

$$
\|f\|_{H^{\varphi}} \sim \|\left(\sum_{j=-\infty}^{\infty} \mid \mathcal{F}^{-1}\left[\left.\tau\left(2^{-j} \cdot\right) \mathcal{F} f\right|^{2}\right)^{\frac{1}{2}} \|_{L^{\varphi}}\right.
$$

Proof. One direction is easy to prove: Simply use the fact that

$$
T f=\sum_{j=-\infty}^{\infty} a_{j} \mathcal{F}^{-1}\left[\tau\left(2^{-j} \cdot\right) \mathcal{F} f\right]=(2 \pi)^{\frac{n}{2}} \sum_{j=-\infty}^{\infty} a_{j} \mathcal{F}^{-1}\left[\tau\left(2^{-j} \cdot\right)\right] * f
$$

is a generalized singular integral operator $T$ with the kernel $K \in L_{\text {loc }}^{1}\left(\mathbb{R}^{n} \times \mathbb{R}^{n} \backslash\right.$ $\left.\left\{(x, x): x \in \mathbb{R}^{n}\right\}\right)$ of order $d_{\varphi}$ with the constant independent of $a=\left\{a_{j}\right\}_{j=-\infty}^{\infty} \in$ $\{-1,0,1\}^{\mathbb{Z}} \cap \ell^{1}(\mathbb{Z})$. More precisely, if we denote by $c_{\alpha, \beta}(T)$ the implicit constant in (4.2), then there exists a constant $C$ independent of $a=\left\{a_{j}\right\}_{j=-\infty}^{\infty} \in\{-1,0,1\}^{\mathbb{Z}} \cap$ $\ell^{1}(\mathbb{Z})$ such that

$$
\|T\|_{L^{2} \rightarrow L^{2}}+c_{\alpha, \beta}(T) \leq C
$$

For the opposite inequality, we will use the $\ell^{2}$-valued extension as in [23]. 


\section{Wavelet characterization}

Based on the fundamental wavelet theory (see [4, 18, 20, 26, 32] for example), we can construct compactly supported functions.

Definition 5.1. Let $s \in \mathbb{N} \cup\{0\}$ be fixed.

1. Choose compactly supported functions $\psi^{l}\left(l=1,2, \ldots, 2^{n}-1\right)$ so that the following conditions are satisfied:

- The functions $\psi^{l}\left(l=1,2, \ldots, 2^{n}-1\right)$ belong to $C^{s+1}\left(\mathbb{R}^{n}\right)$. In addition, they are real-valued and compactly supported with

$$
\operatorname{supp}\left(\psi^{l}\right) \subset[0,2 N-1]^{n}
$$

for some $N \in \mathbb{N}$.

- We write $\psi_{j, k}^{l} \equiv 2^{\frac{j n}{2}} \psi^{l}\left(2^{j} \cdot-k\right)$ for $j \in \mathbb{Z}$ and $k \in \mathbb{Z}^{n}$. Then the system

$$
\left\{\psi_{j, k}^{l}: k \in \mathbb{Z}^{n}, j \in \mathbb{Z}, l=1,2, \ldots, 2^{n}-1\right\}
$$

is an orthonormal basis of $L^{2}\left(\mathbb{R}^{n}\right)$.

In the above it should be noted

$$
\operatorname{supp}\left(\psi_{j, k}^{l}\right) \subset \prod_{m=1}^{n}\left[2^{-j} k_{m}, 2^{-j}\left(k_{m}+2 N-1\right)\right] \subset 4 N Q_{j, k} .
$$

Recall that $Q_{j, k}$ is a dyadic cube by (1.2). According to [32], $\psi^{l} \perp \mathcal{P}_{s+1}(\mathbb{R})$ when $n=1$. This fact readily extends to higher dimensions by the tensor product: $\psi^{l} \perp \mathcal{P}_{s+1}\left(\mathbb{R}^{n}\right)$ for any $n \in \mathbb{N}$.

We also define $\chi_{j, k} \equiv 2^{\frac{j n}{2}} \chi_{Q_{j, k}}$ for $j \in \mathbb{Z}$ and $k=\left(k_{1}, k_{2}, \ldots, k_{n}\right) \in \mathbb{Z}^{n}$, where $Q_{j, k}$ is the dyadic cube given by (1.2). Then using the $L^{2}$-inner product $\langle\cdot, \cdot\rangle$, we define the square function $W f$ by

$$
W f \equiv\left(\sum_{l=1}^{2^{n}-1} \sum_{j=-\infty}^{\infty} \sum_{k \in \mathbb{Z}^{n}}\left|\left\langle f, \psi_{j, k}^{l}\right\rangle \chi_{j, k}\right|^{2}\right)^{\frac{1}{2}}
$$

at least for $f \in L^{2}\left(\mathbb{R}^{n}\right)$.

We now move on to the investigation of the wavelet characterization of $H^{\varphi}\left(\mathbb{R}^{n}\right)$. In a similar way to the proof of Theorem 4.4, we can show the following:

Lemma 5.2. Let $\varphi \in \mathbb{A}_{\infty}\left(\mathbb{R}^{n}\right)$ satisfy $0<i(\varphi) \leq I(\varphi)<\infty$. Then there exists a positive constant $C=C_{\psi}$ depending on $\psi^{l}$ such that

$$
\left\|\left(\sum_{j=-\infty}^{\infty}\left|2^{j n} \psi^{l}\left(2^{j} \cdot\right) * f\right|^{2}\right)^{\frac{1}{2}}\right\|_{L^{\varphi}} \leq C\|f\|_{H^{\varphi}}
$$

for all $f \in H^{\varphi}\left(\mathbb{R}^{n}\right) \cap L^{2}\left(\mathbb{R}^{n}\right)$. 
We set

$$
\psi_{j, \nu}^{l, * *}(f)(x)=\sup _{y \in \mathbb{R}^{n}} \frac{\left|f * \psi^{l}\left(2^{j}(x-y)\right)\right|}{\left(1+2^{j}|y|\right)^{n \nu}} \quad\left(x \in \mathbb{R}^{n}\right) .
$$

As is pointed out in [5, Proof of Proposition 2.21] and [9, p. 271], we have

$$
\psi_{j, \nu}^{l, * *}(f)(x) \lesssim M\left[\left|2^{j n} f * \psi^{l}\left(2^{j} \cdot\right)\right|^{\frac{1}{\nu}}\right](x)^{\nu}=M^{(\nu)}\left[2^{j n} f * \psi^{l}\left(2^{j} \cdot\right)\right](x) \quad\left(x \in \mathbb{R}^{n}\right)
$$

for all $f \in H^{\varphi}\left(\mathbb{R}^{n}\right) \cap L^{2}\left(\mathbb{R}^{n}\right)$.

Corollary 5.3. Let $\varphi \in \mathbb{A}_{\infty}\left(\mathbb{R}^{n}\right)$ satisfy $0<i(\varphi) \leq I(\varphi)<\infty$. Suppose that the parameter $\nu$ satisfies $\nu \in(q(\varphi) / i(\varphi), \infty)$. Then

$$
\left\|\left(\sum_{l=1}^{2^{n}-1} \sum_{j=-\infty}^{\infty} \psi_{j, \nu}^{l, * *}(f)^{2}\right)^{\frac{1}{2}}\right\|_{L^{\varphi}} \lesssim\|f\|_{H^{\varphi}}
$$

for all $f \in H^{\varphi}\left(\mathbb{R}^{n}\right)$.

We will use Corollary 5.3 to justify the definition of $\left\langle f, \psi_{j, k}^{l}\right\rangle$.

Lemma 5.4. Let $\varphi \in \mathbb{A}_{\infty}\left(\mathbb{R}^{n}\right)$ satisfy $0<i(\varphi) \leq I(\varphi)<\infty$. Assume that $s>d_{\varphi}$, where $d_{\varphi}$ is given by (2.6). Let $f \in H^{\varphi}\left(\mathbb{R}^{n}\right) \cap L^{2}\left(\mathbb{R}^{n}\right)$. Then $\|W f\|_{L^{\varphi}} \lesssim\|f\|_{H^{\varphi}}$. In particular, the mapping $f \mapsto\left\langle f, \psi_{j, k}^{l}\right\rangle$ extends to a bounded linear functional on $H^{\varphi}\left(\mathbb{R}^{n}\right)$ for each $j, k, l$.

Proof. Fix $x \in \mathbb{R}^{n}$. We write $\tilde{\psi}^{l} \equiv \overline{\psi^{l}(-\cdot)}$ for each $l=1,2, \ldots, 2^{n}-1$. If $f \in$ $H^{\varphi}\left(\mathbb{R}^{n}\right) \cap L^{2}\left(\mathbb{R}^{n}\right)$, then Fu and Yang showed

$$
\sum_{k \in \mathbb{Z}^{n}}\left|\left\langle f, \psi_{j, k}^{l}\right\rangle\right|^{2} \chi_{4 N Q_{j, k}}(x) \lesssim \sum_{k \in \mathbb{Z}^{n}}\left[\sup _{y \in Q_{j, k}}\left|\tilde{\psi}_{j}^{l} * f(y)\right|\right] \chi_{4 N \chi_{Q_{j}, k}}(x) \lesssim \psi_{j, \nu}^{l, * *}(f)(x)^{2}
$$

in the course of the proof of $[5$, Theorem 1.9]. Hence

$$
W f(x) \lesssim\left(\sum_{l=1}^{2^{n}-1} \sum_{j=-\infty}^{\infty} \psi_{j, \nu}^{l, * *}(f)(x)^{2}\right)^{\frac{1}{2}}
$$

Thus, by Corollary 5.3, we obtain $W f \in L^{\varphi}\left(\mathbb{R}^{n}\right)$ together with the estimate $\left|\left\langle f, \psi_{j, k}^{l}\right\rangle\right| \lesssim\|W f\|_{L^{\varphi}} \lesssim\|f\|_{H^{\varphi}}$.

Lemma 5.4 also justifies the definition of $W f$. We further investigate the property of $W f$.

Lemma 5.5. Let $\varphi \in \mathbb{A}_{\infty}\left(\mathbb{R}^{n}\right)$ satisfy $0<i(\varphi) \leq I(\varphi)<\infty$. Assume that

$$
s \in \mathbb{Z} \cap\left(n \max \left(\frac{1}{2}, \frac{q(\varphi)}{i(\varphi)}\right)-n-1, \infty\right) \cap[0, \infty) .
$$

(1) Let $f \in H^{\varphi}\left(\mathbb{R}^{n}\right)$. Then $\|W f\|_{L^{\varphi}} \lesssim\|f\|_{H^{\varphi}}$ with the implicit constant independent of $f$. 
(2) If $f \in L^{2}\left(\mathbb{R}^{n}\right)$ satisfies $W f \in L^{\varphi}\left(\mathbb{R}^{n}\right)$, then $\|f\|_{H^{\varphi}} \lesssim\|W f\|_{L^{\varphi}}$ with the implicit constant independent of $f$.

Proof.

1. This is included in Lemma 5.4.

2. Let $f \in L^{2}\left(\mathbb{R}^{n}\right)$. Then we expand

$$
f=\sum_{l=1}^{2^{n}-1} \sum_{j=-\infty}^{\infty} \sum_{k \in \mathbb{Z}^{n}}\left\langle f, \psi_{j, k}^{l}\right\rangle \psi_{j, k}^{l}
$$

Fix $\varepsilon>0$ and $\kappa>\max \left(\frac{1}{2}, \frac{q(\varphi)}{i(\varphi)}\right)$ so that

$$
\kappa n+2 \varepsilon-n-s-1<0
$$

later. Write

$$
E\left(j, j^{\prime}\right)=n \min \left(j, j^{\prime}\right)-\frac{n}{2} j-(s+1)\left|j-j^{\prime}\right|+(\kappa n+\varepsilon) \max \left(j-j^{\prime}, 0\right)
$$

for $j, j^{\prime} \in \mathbb{Z}$. Arithmetic shows

$$
E\left(j, j^{\prime}\right)-\frac{n}{2} j+\varepsilon\left|j-j^{\prime}\right|= \begin{cases}(\kappa n+2 \varepsilon-n-s-1)\left(j-j^{\prime}\right) & \left(j \geq j^{\prime}\right) \\ (\varepsilon-s-1)\left(j^{\prime}-1\right) & \left(j^{\prime}>j\right) .\end{cases}
$$

We will use $M^{(\eta)}$ given by $(2.5)$.

We observe

$$
\begin{aligned}
\mathcal{F}^{-1}\left[\tau\left(2^{-j^{\prime}} \cdot\right)\right] * \psi_{j, k}^{l}(x) & =2^{j^{\prime} n} \mathcal{F}^{-1} \tau\left(2^{j^{\prime}} \cdot\right) *\left[2^{\frac{j n}{2}} \psi^{l}\left(2^{j} \cdot-k\right)\right](x) \\
& =2^{-\frac{j n}{2}} 2^{j^{\prime} n} \mathcal{F}^{-1} \tau\left(2^{j^{\prime}} \cdot\right) *\left[2^{j n} \psi^{l}\left(2^{j} \cdot\right)\right]\left(x-2^{-j} k\right) .
\end{aligned}
$$

Let $\lambda \gg 1$. Thanks to Lemma 2.19, since $\psi^{l} \perp \mathcal{P}_{s}\left(\mathbb{R}^{n}\right) \cap C^{s+1}\left(\mathbb{R}^{n}\right)$,

$$
\left|\mathcal{F}^{-1}\left[\tau\left(2^{-j^{\prime}} \cdot\right) \mathcal{F} \psi_{j, k}^{l}\right](x)\right| \lesssim \frac{2^{-\frac{j n}{2}+\min \left(j, j^{\prime}\right) n-\left|j-j^{\prime}\right|(s+1)}}{\left(1+2^{\min \left(j, j^{\prime}\right)}\left|x-2^{-j} k\right|\right)^{\lambda}} .
$$

Using (5.2) and [26, Lemma 4.2], we obtain

$$
\begin{aligned}
\left|\mathcal{F}^{-1}\left[\tau\left(2^{-j^{\prime}} .\right) \mathcal{F} f\right]\right| & \lesssim \sum_{l=1}^{2^{n}-1} \sum_{j=-\infty}^{\infty} \sum_{k \in \mathbb{Z}^{n}} \frac{\left|\left\langle f, \psi_{j, k}^{l}\right\rangle\right| 2^{n \min \left(j, j^{\prime}\right)-\frac{n}{2} j-(s+1)\left|j-j^{\prime}\right|}}{\left(1+2^{\min \left(j, j^{\prime}\right)}\left|\cdot-2^{-j} k\right|\right)^{\kappa n+\varepsilon}} \\
& \lesssim \sum_{l=1}^{2^{n}-1} \sum_{j=-\infty}^{\infty} \sum_{k \in \mathbb{Z}^{n}} \frac{\left|\left\langle f, \psi_{j, k}^{l}\right\rangle\right| 2^{E\left(j, j^{\prime}\right)}}{\left(1+2^{j}\left|\cdot-2^{-j} k\right|\right)^{\kappa n+\varepsilon}} \\
& \lesssim \sum_{l=1}^{2^{n}-1} \sum_{j=-\infty}^{\infty} M^{(1 / \kappa)}\left[\sum_{k \in \mathbb{Z}^{n}}\left|\left\langle f, \psi_{j, k}^{l}\right\rangle\right| 2^{E\left(j, j^{\prime}\right)} \chi_{Q_{j k}}\right] .
\end{aligned}
$$


Thus,

$$
\begin{aligned}
& \left|\mathcal{F}^{-1}\left[\tau\left(2^{-j^{\prime}} \cdot\right) \mathcal{F} f\right]\right|^{2} \\
& \lesssim \sum_{l=1}^{2^{n}-1} \sum_{j=-\infty}^{\infty}\left(M^{(1 / \kappa)}\left[\sum_{k \in \mathbb{Z}^{n}}\left|\left\langle f, \psi_{j, k}^{l}\right\rangle\right| 2^{E\left(j, j^{\prime}\right)+\varepsilon\left|j-j^{\prime}\right|} \chi_{Q_{j k}}\right]\right)^{2}
\end{aligned}
$$

by Hölder's inequality. We will take the $\ell^{2}$-norm of both sides to have

$$
\begin{aligned}
& \left\|\left(\sum_{j^{\prime}=-\infty}^{\infty}\left|\mathcal{F}^{-1}\left[\tau\left(2^{-j^{\prime}} \cdot\right) \mathcal{F} f\right]\right|^{2}\right)^{\frac{1}{2}}\right\| \\
& \lesssim\left\|\left(\sum_{l=1}^{2^{n}-1} \sum_{j=-\infty}^{\infty} \sum_{j^{\prime}=-\infty}^{\infty} M^{(1 / \kappa)}\left[\sum_{k \in \mathbb{Z}^{n}}\left|\left\langle f, \psi_{j, k}^{l}\right\rangle\right| 2^{E\left(j, j^{\prime}\right)+\varepsilon\left|j-j^{\prime}\right|} \chi_{Q_{j k}}\right]^{2}\right)^{\frac{1}{2}}\right\|_{L^{\varphi}} \\
& =\left\|\left(\sum_{l=1}^{2^{n}-1} \sum_{j=-\infty}^{\infty} \sum_{j^{\prime}=-\infty}^{\infty} M\left[\left|\sum_{k \in \mathbb{Z}^{n}}\right|\left\langle f, \psi_{j, k}^{l}\right\rangle\left|2^{E\left(j, j^{\prime}\right)+\varepsilon\left|j-j^{\prime}\right|} \chi_{Q_{j k}}\right|^{\frac{1}{\kappa}}\right]^{2 \kappa}\right)^{\frac{1}{2 \kappa}}\right\|_{L^{\varphi\left(\cdot, \cdot(\cdot)^{\kappa}\right)}}^{\kappa}
\end{aligned}
$$

Recall that

$$
\begin{gathered}
2 \kappa>1, \quad E\left(j, j^{\prime}\right)-\frac{j n}{2}+\varepsilon\left|j-j^{\prime}\right|<0, \\
i\left(\varphi\left(\cdot,(\cdot)^{\kappa}\right)\right)=\kappa i(\varphi)>q\left(\varphi\left(\cdot,(\cdot)^{\kappa}\right)\right)=q(\varphi) .
\end{gathered}
$$

By the Fefferman-Stein vector-valued inequality (see Lemma 2.11), we obtain

$$
\begin{aligned}
& \left\|\left(\sum_{j^{\prime}=-\infty}^{\infty}\left|\mathcal{F}^{-1}\left[\tau\left(2^{-j^{\prime}} .\right) \mathcal{F} f\right]\right|^{2}\right)^{\frac{1}{2}}\right\|_{L^{\varphi}} \\
& \lesssim\left\|\left(\sum_{l=1}^{2^{n}-1} \sum_{j=-\infty}^{\infty} \sum_{j^{\prime}=-\infty}^{\infty}\left[\sum_{k \in \mathbb{Z}^{n}}\left|\left\langle f, \psi_{j, k}^{l}\right\rangle\right| 2^{E\left(j, j^{\prime}\right)+\varepsilon\left|j-j^{\prime}\right|} \chi_{Q_{j k}}\right]^{2}\right)^{\frac{1}{2}}\right\|_{L^{\varphi}} \\
& \sim\left\|\left(\sum_{l=1}^{2^{n}-1} \sum_{j=-\infty}^{\infty}\left[\sum_{k \in \mathbb{Z}^{n}}\left|\left\langle f, \psi_{j, k}^{l}\right\rangle\right| 2^{\frac{n}{2} j} \chi_{Q_{j k}}\right]^{2}\right)^{\frac{1}{2}}\right\|_{L^{\varphi}} \\
& =\|W f\|_{L^{\varphi}} .
\end{aligned}
$$

Thus, thanks to Theorem 4.4, we obtain the desired result.

Theorem 5.6. Let $\varphi \in \mathbb{A}_{\infty}\left(\mathbb{R}^{n}\right)$ satisfy $0<i(\varphi) \leq I(\varphi)<\infty$. Assume that

$$
s \in \mathbb{Z} \cap\left(n \max \left(\frac{1}{2}, \frac{q(\varphi)}{i(\varphi)}\right)-n-1, \infty\right) \cap[0, \infty) .
$$

Assume either one of the following: 
(i) $f \in H^{\varphi}\left(\mathbb{R}^{n}\right)$.

(ii) $f \in L^{2}\left(\mathbb{R}^{n}\right)$ and $W f \in L^{\varphi}\left(\mathbb{R}^{n}\right)$.

Then $\|W f\|_{L^{\varphi}} \sim\|f\|_{H^{\varphi}}$ with the implicit constant independent of $f$.

Proof. Lemma 5.5 yields the desired result under assumption $(i i)$. Let us assume (i). If $f \in H^{\varphi}\left(\mathbb{R}^{n}\right)$, then we can find a sequence $\left\{f_{j}\right\}_{j=1}^{\infty} \subset H^{\varphi}\left(\mathbb{R}^{n}\right) \cap L^{2}\left(\mathbb{R}^{n}\right)$ that converges to $f$ in $H^{\varphi}\left(\mathbb{R}^{n}\right)$. In view of Lemma 5.5(1),Wf= $\lim _{j \rightarrow \infty} W f_{j}$ in $L^{\varphi}\left(\mathbb{R}^{n}\right)$. Thus, $\lim _{j \rightarrow \infty}\left\|W f_{j}\right\|_{L^{\varphi}}=\|W f\|_{L^{\varphi}}$. Since $\left\{f_{j}\right\}_{j=1}^{\infty}$ converges to $f$ in $H^{\varphi}\left(\mathbb{R}^{n}\right),\|f\|_{H^{\varphi}}=$ $\lim _{j \rightarrow \infty}\left\|f_{j}\right\|_{H^{\varphi}}$. Meanwhile, Lemma 5.5(2) yields $\lim _{j \rightarrow \infty}\left\|f_{j}\right\|_{H^{\varphi}} \lesssim \lim _{j \rightarrow \infty}\left\|W f_{j}\right\|_{L^{\varphi}}$.

\section{Generalized Marcinkiewicz integral operators acting on Musielak-Orlicz Hardy spaces}

This section is oriented to another application of the atomic characterization. Let $0<\rho<n$ and $1<q<\infty$. Let also $\Omega \in C^{1}\left(S^{n-1}\right)$ satisfy

$$
\int_{S^{n-1}} \Omega(\omega) d \sigma(\omega)=0
$$

The generalized Marcinkiewicz operator is defined by

$$
\mu_{\Omega, \rho, q} f(x)=\left(\int_{0}^{\infty}\left|\frac{1}{t^{\rho}} \int_{B(t)} f(x-y) \frac{\Omega(y /|y|)}{|y|^{n-\rho}} d y\right|^{q} \frac{\mathrm{d} t}{t}\right)^{\frac{1}{q}},
$$

where $B(r)$ denotes the open ball centered at the origin for $r>0$. According to [27, Theorem 1], we have

$$
\left\|\mu_{\Omega, \rho, q} f\right\|_{L^{p}} \lesssim\|f\|_{\dot{F}_{p, q}^{0}}
$$

if $1<p<\infty$.

As in $[2$, p. 565],

$$
\chi_{\mathbb{R}^{n} \backslash 3 n Q}(x) \mu_{\Omega, \rho, q} a(x) \lesssim \frac{1}{\left\|\chi_{Q}\right\|_{L^{\varphi}}} M \chi_{Q}(x)^{1+\frac{1}{n q}} \quad\left(x \in \mathbb{R}^{n}\right)
$$

as long as $a$ is a $(\varphi, \infty)$-atom supported on $3 Q$. Using (6.2) with $q=2$, we learn that $\mu_{\Omega, \rho, 2}$ is bounded on $L^{p}\left(\mathbb{R}^{n}\right)$ for all $1<p<\infty$. A direct consequence of this fact is that

$$
\left\|\chi_{3 n Q} \cdot \mu_{\Omega, \rho, q} a\right\|_{L^{u}} \lesssim \frac{|Q|^{1 / u}}{\left\|\chi_{Q}\right\|_{L^{\varphi}}} \sim \frac{|Q|^{1 / u}}{\left\|\chi_{3 n Q}\right\|_{L^{\varphi}}}
$$

for all $1<u<\infty$.

Theorem 6.1. Let $0<\rho<n$ and $\Omega \in C^{1}\left(S^{n-1}\right)$ satisfy (6.1). Let $\varphi \in \mathbb{A}_{\infty}\left(\mathbb{R}^{n}\right)$ satisfy

$$
\frac{2 n}{2 n+1} q(\varphi)<i(\varphi) \leq I(\varphi)<\infty .
$$

Then $\mu_{\Omega, \rho, 2}$, initially defined on $L^{2}\left(\mathbb{R}^{n}\right)$, extends to a bounded linear operator from $H^{\varphi}\left(\mathbb{R}^{n}\right)$ to $L^{\varphi}\left(\mathbb{R}^{n}\right)$. 
Proof. Let $f \in H^{\varphi}\left(\mathbb{R}^{n}\right) \cap L^{2}\left(\mathbb{R}^{n}\right)$. It suffices to show that

$$
\left\|\mu_{\Omega, \rho, 2} f\right\|_{L^{\varphi}} \lesssim\|f\|_{H^{\varphi}}
$$

with the implicit constant independent of $f$.

We consider the atomic characterization of $f$ :

$$
f=\sum_{j=1}^{\infty} \kappa_{j} a_{j}
$$

where the convergence takes place in $L^{2}\left(\mathbb{R}^{n}\right) \cap H^{\varphi}\left(\mathbb{R}^{n}\right),\left\{\kappa_{j}\right\}_{j=1}^{\infty}$ is a sequence of nonnegative numbers, $\left\{\left(a_{j}, Q_{j}\right)\right\}_{j=1}^{\infty} \subset \mathcal{A}(\varphi, \infty)$ and $\mathcal{A}\left(\left\{\kappa_{j}\right\}_{j=1}^{\infty},\left\{Q_{j}\right\}_{j=1}^{\infty}\right) \lesssim\|f\|_{H^{\varphi}}<$ $\infty$. Then we have

$$
\mu_{\Omega, \rho, 2} f \lesssim \sum_{j=1}^{\infty} \kappa_{j} \chi_{3 n Q_{j}} \mu_{\Omega, \rho, 2} a_{j}+\sum_{j=1}^{\infty} \frac{\kappa_{j}}{\left\|\chi_{Q_{j}}\right\|_{L^{\varphi}}}\left(M \chi_{Q_{j}}\right)^{1+\frac{1}{2 n}}
$$

using (6.3). If we take the $L^{\varphi}$-norm and use Lemmas 2.11 and 2.16 together with (6.4), we obtain

$$
\left\|\mu_{\Omega, \rho, 2} f\right\|_{L^{\varphi}} \lesssim \mathcal{A}\left(\left\{\kappa_{j}\right\}_{j=1}^{\infty},\left\{Q_{j}\right\}_{j=1}^{\infty}\right)
$$

as required.

In the case of $\varphi(x, t)=t^{p}$ with $\frac{2 n}{2 n+1}<p \leq 1$, Theorem 6.1 overlaps a result in [2]. As a special case of Theorem 6.1, we have the following:

Corollary 6.2. Let $0<\rho<n$ and $\Omega \in C^{1}\left(S^{n-1}\right)$ satisfy (6.1). The operator $\mu_{\Omega, \rho, 2}$, initially defined on $L^{2}\left(\mathbb{R}^{n}\right)$, extends to a bounded linear operator from $H^{p}\left(\mathbb{R}^{n}\right)$ to $L^{p}\left(\mathbb{R}^{n}\right)$ whenever $\frac{2 n}{2 n+1}<p \leq 1$.

\section{Acknowledgements}

This work was partly supported by Osaka City University Advanced Mathematical Institute: MEXT Joint Usage/Research Center on Mathematics and Theoretical Physics JPMXP0619217849.

Mitsuo Izuki was partially supported by Grand-in-Aid for Scientific Research (C), No. 15K04928, for Japan Society for the Promotion of Science. Eiichi Nakai was partially supported by Grand-in-Aid for Scientific Research (B), No. 15H03621, for Japan Society for the Promotion of Science. Yoshihiro Sawano was partially supported by Grand-in-Aid for Scientific Research (C), No. 19K03546, for Japan Society for the Promotion of Science. We started this work during the stay of the first and the third authors in Ibaraki University.

\section{References}

[1] A. Akbulut, V.S. Guliyev, T. Noi and Y. Sawano, Generalized Hardy-Morrey spaces, Z. Anal. Anwend., 36 (2017), no. 2, 129-149. 
[2] K. Asami, Nonsmooth decomposition and the Marcinkiewicz integral, International Journal of Applied Mathematics, 30, No. 6 2017, 547-568. [2]

[3] T. Batbold and Y. Sawano, Decompositions for local Morrey spaces, Eurasian Math. J., 5 (2014), no. 3, 9-45. [3, 30, 31]

[4] I. Daubechies, Orthonormal basis of compactly supported wavelets, Comm. Pure Appl. Math. 41 (1988), 909-996. [2] [26]

[5] X. Fu and D. Yang, Wavelet characterizations of Musielak-Orlicz Hardy space, Banach J. Math. Anal. 12 (2018), no. 4, 1017-1046. [2, 6, 27]

[6] J. García-Cuerva and J. M. Martell, Wavelet characterization of weighted spaces, J. Geom. Anal. 11 (2001), no. 2, 241-264. [2]

[7] L. Grafakos, Modern Fourier Analysis. Graduate texts in mathematics; 250, New York, Springer, 2014. [3, 5, 16]

[8] V.S. Guliyev, S.G. Hasanov and Y. Sawano, Decompositions of local Morreytype spaces, Positivity, 21, no. 3, 1223-1252 (2017) [2]

[9] E. Hernández and G. Weiss, A First Course on Wavelets, CRC Press, Boca Raton, FL, 1996. [27]

[10] K.-P. Ho, Atomic decomposition of Hardy-Morrey spaces with variable exponents, Annales Academiae Scientiarum Fennicae. Mathematica Volume 40, Issue $1,31-62$. [2]

[11] L. Huang, J. Liu, D. Yang and W. Yuan, Atomic and Littlewood-Paley characterizations of anisotropic mixed-norm Hardy spaces and their applications, J. Geom. Anal. 29 (2019), 1991-2067. [2]

[12] M. Izuki, Boundedness of sublinear operators on Herz spaces with variable exponent and application to wavelet characterization, Anal. Math. 36 (2010), no. $2,33-50 .[2]$

[13] M. Izuki, E. Nakai and Y. Sawano, Wavelet characterization and modular inequalities for weighted Lebesgue spaces with variable exponent, Ann. Acad. Sci. Fenn. Math. 40 (2015), no. 2, 551-571. [2]

[14] T. Iida, Y. Sawano and H. Tanaka, Atomic Decomposition for Morrey Spaces, Z. Anal. Anwend. 33 (2014), no. 2, 149-170. [2]

[15] V. Kokilashvili and M. Krbec, Weighted Inequalities in Lorentz and Orlicz Spaces, River Edge, NJ: World Scientific Publishing, 1991. [8]

[16] T.S. Kopaliani, Greediness of the wavelet system in $L^{p(t)}(\mathbb{R})$ spaces, East J. Approx. 14 (2008), no. 1, 59-67. [2]

[17] L.D. Ky, New Hardy spaces of Musielak-Orlicz type and boundedness of sublinear operators. Integral Equations Operator Theory 78, no. 1 (2014), 115150. $[1,2,3,4,5,6,17,23,24]$ 
[18] P.G. Lemarié-Rieusset and G. Malgouyres, Support des fonctions de base dans une analyse multi-résolution, C. R. Acad. Sci. Paris 313 (1991), 377-380. [26]

[19] H.P. Liu, The wavelet characterization of the space weak $H^{1}$, Studia Math. 103 (1992), no. 1, 109-117. [2]

[20] Y. Meyer, Wavelets and Operators, Cambridge Univ. Press, Cambridge, 1992. $[2,26]$

[21] F.-Y. Maeda, Y. Sawano; T. Shimomura, Some norm inequalities in MusielakOrlicz spaces, Ann. Acad. Sci. Fenn. Math. 41 (2016), no. 2, 721-744. [2, 8, 9]

[22] E. Nakai and Y. Sawano, Orlicz -Hardy spaces and their duals, Sci. China Math. 57 (2014), no. 5, 903-962. [8]

[23] E. Nakai and Y. Sawano, Hardy spaces with variable exponents and generalized Campanato spaces, J. Funct. Anal. 262 (2012), 3665-3748. [2, 3, 14, 17, $19,20,24,25]$

[24] E. Nakai and K. Yabuta, Pointwise multipliers for functions of bounded mean oscillation, J. Math. Soc. Japan 37 (1985), 207-218. [2]

[25] Y. Sawano, Atomic decompositions of Hardy spaces with variable exponents and its application to bounded linear operators. Integral Equ. Oper. Theory 77 (2013), 123-148. [2]

[26] Y. Sawano, Theory of Besov Spaces, Development in Mathematics 56, Springer, Singapore, 2018. [3, 5, 15, 16, 17, 26, 28]

[27] Y. Sawano and K. Yabuta, Fractional type Marcinkiewicz integral operators associated to surfaces, J. Inequal. Appl., 2014 (2014), art. $\sharp 232,29$ pp. [30]

[28] E.M. Stein, Harmonic Analysis, real-variable methods, orthogonality, and oscillatory integrals, Princeton University Press, Princeton, NJ, 1993. [15, 16]

[29] D. Yang, Y. Liang and L.D. Ky, Real-variable theory of Musielak-Orlicz Hardy spaces. Lecture Notes in Mathematics, 2182. Springer, Cham, 2017. xiii+466 pp. $[2,4,10]$

[30] X. Yan, D. Yang, W. Yuan and C. Zhuo, Variable weak Hardy spaces and their applications, J. Funct. Anal., 271, Issue 10, 2822-2887. [2]

[31] D. Yang, C. Zhuo, and E. Nakai, Characterizations of variable exponent Hardy spaces via Riesz transforms, Rev. Mat. Complut. 29 (2016), no. 2, 245-270. $[2]$

[32] P. Wojtaszczyk, A Mathematical Introduction to Wavelets. Cambridge University Press, Cambridge, 1997. [26] 
[33] S. Wu, A wavelet characterization for weighted Hardy spaces, Rev. Mat. Iberoam. 8 (1992), no. 3, 329-349. [2]

Mitsuo Izuki,

Faculty of Liberal Arts and Sciences,

Tokyo City University,

1-28-1, Tamadutsumi Setagaya-ku Tokyo 158-8557, Japan.

E-mail: izuki@tcu.ac.jp

Eiichi Nakai,

Department of Mathematics,

Ibaraki University,

Mito, Ibaraki 310-8512, Japan.

E-mail: eiichi.nakai.math@vc.ibaraki.ac.jp

Yoshihiro Sawano,

Department of Mathematical Science,

Chuo University,

Kasuga, Bunkyo-ku 112-8551, Tokyo, Japan.

$+$

People's Friendship University of Russia.

E-mail: yoshihiro-sawano@celery.ocn.ne.jp 